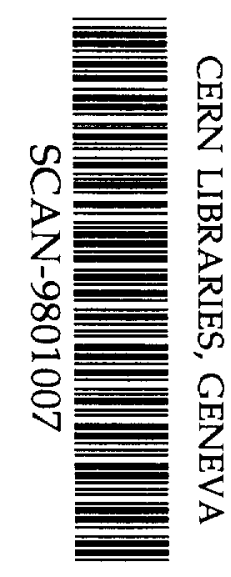

swg803

\title{
FISSION THROUGH QUASI-MOLECULAR SHAPES AND
} FRAGMENTATION

\author{
G. Royer \\ To be published in : \\ "Heavy Elements and Related Phenomena" \\ (Editors : Raj K. Gupta and W. Greiner) \\ Rapport Interne SUBATECH 97-23
}




\title{
Fission through quasi-molecular shapes and fragmentation
}

\author{
G. Royer \\ Laboratoire de Physique Subatomique et des Technologies Associées, UMR : IN2P3/ CNRS, \\ Université et Ecole des Mines, 4 rue A. Kastler, 44307 Nantes Cedex 03, France
}

\begin{abstract}
The decay channel leading to two or several tangent and later separated spherical fragments assuming volume conservation and neck formation while keeping quasi-spherical ends has been investigated. The potential energy has been determined within a generalized liquid-drop model including a proximity energy term, the decay asymmetry, an accurate nuclear radius and the temperature dependence. The calculated fission barrier characteristics, half-lives of radioactive nuclei emitting heavy clusters, fragment kinetic energies, critical angular momenta of light nuclei and rotating super and hyperdeformed state properties are in agreement with the available experimental data. The formation of evanescent quasi-toroidal systems and fragmentation with emission in a plane seem possible in very heavy-ion collisions at intermediate energies.
\end{abstract}

\section{Introduction}

New observed phenomena like cold and asymmetric fission of ${ }^{252} \mathrm{Cf}[1-2]$, cluster radioactivity [3-4], formation of nuclear molecules in ${ }^{24} \mathrm{Mg}$ [5], asymmetric fission of intermediate mass nuclei [6] and quasi-fission of heavy dinuclear systems [7] have renewed interest in investigating the fusion-like fission valley which leads rapidly to two touching spherical fragments and quasi-molecular shapes. Furthermore, the rotating super and hyperdeformed states as well as the very heavy and possible superheavy elements are and will be formed in heavy-ion collisions for which the starting configuration is two close quasi-spherical nuclei. In more violent heavy-ion reactions (a few tens of $\mathrm{MeV}$ per nucleon), hot and compressed nuclear systems are produced and their fragmentation in several relatively cold and spherical intermediate mass fragments is observed [8-9].

The earlier fission studies assumed that the balance between the repulsive Coulomb forces and attractive surface tension forces governs the evolution of the nuclear shapes [10-11]. The often used radius development in Legendre polynomials led to elongated one-body configurations which have been able to explain the bulk of our knowledge on nuclear fission. Nevertheless, this development fails to reproduce strongly distorted configurations [12] with deep and narrow necks and, consequently, it is difficult to link the two sheets of the potential-energy surface corresponding respectively to one-body shapes and to two separaied fragments [13]. This problem remains in the microscopic approaches [14-15].

Later on, the inefficiency of the pure Coulomb barrier to reproduce the fusion cross sections has led to the introduction of a proximity term [16-17] in the development of the liquid drop model energy in order to smoothly describe the transition from two 
almost spherical nuclei to one-body shapes in properly taking into account the finiterange effects of the nuclear force in the gap or crevice between the incoming nuclei. As an example, for two touching spherical heavy nuclei, the proximity energy reaches around $-40 \mathrm{MeV}$ while the fission barrier height of the compound nucleus is only 5-10 $\mathrm{MeV}$. Dynamical studies have pointed out the possibility of fission processes through compact shapes [18] and, at least, cluster emission, cold and asymmetric fission and fragmentation are exit modes exploring these distorted shapes where the necks are not shallow. Consequently, it is important to study the role that the proximity forces might play also in the nuclear fission process.

The study of the compatibility with the available data of a decay through quasimolecular shapes is presented here, starting from the viewpoint that a degeneracy may exist between the energy of elongated shapes found by the liquid drop model without proximity energy term and the energy of compact and necked shapes for which the lowering of the potential energy is due to the proximity interaction in the neck. A generalized liquid drop model is firstly defined in section 2 while in section 3 the shape sequence selected to describe the transition from one sphere to two tangent spheres is displayed. The ability of these adopted liquid drop model and shape sequence to reproduce the fusion data has been checked [19-20]. The main characteristics of the exit channel via these quasi-molecular shapes are compared with symmetric and asymmetric fission barrier data in sections 4 and 5 , with new results on cluster radioactivity in section 6 and with fragment kinetic energies in section 7 . The existence of rotating super and hyperdeformed states and the critical angular momentum that a nucleus is able to sustain against centrifugal forces in this peculiar deformation valley are investigated in section 8 . The shape sequence has been generalized to study the ternary fission and results are presented in section 9 . Finally, the temperature effects have been introduced in section 10 and the rapid fragmentation process with emission in a plane or in the whole space has been compared with the formation of toroids and bubbles in section 11 .

\section{Generalized liquid-drop model}

For an arbitrary deformed nucleus, the macroscopic total energy is defined as [2123] :

$$
E=E_{R L D M}+E_{N}
$$

where $E_{R L D M}$ and $E_{N}$ are respectively the rotational liquid-drop model energy and the nuclear proximity energy [16-17]. Constant density and volume conservation are assumed.

$$
E_{R L D M}=E_{V}+E_{S}+E_{C}+E_{R o t} .
$$

For one-body shapes, the volume $E_{V}$, surface $E_{S}$ and Coulomb $E_{C}$ energies are given by:

$$
\begin{gathered}
E_{V}=-a_{v}\left(1-k_{v} I^{2}\right) A, \\
E_{S}=a_{s}\left(1-k_{s} I^{2}\right) A^{2 / 3}\left(S / 4 \pi R_{0}^{2}\right), \\
E_{C}=0.6 e^{2}\left(Z^{2} / R_{0}\right) \times 0.5 \int\left(V(\theta) / V_{0}\right)\left(R(\theta) / R_{0}\right)^{3} \sin \theta d \theta
\end{gathered}
$$

where $A, Z$ and $I=(N-Z) / A$ are the mass, charge and relative neutron excess of the compound nucleus. $V(\theta)$ is the electrostatic potential at the surface of the shape and $V_{0}$ the surface potential of the sphere. The volume and surface coefficients $a_{v}, a_{s}$ and the effective sharp radius $R_{0}$ have been chosen as : 


$$
\begin{gathered}
a_{v}(T)=15.494\left(1+0.00337 T^{2}\right) \mathrm{MeV} \\
a_{s}(T)=17.9439(1+1.5 T / 17)(1-T / 17)^{3 / 2} \mathrm{MeV} \\
R_{0}(T)=\left(1.28 A^{1 / 3}-0.76+0.8 A^{-1 / 3}\right)\left(1+0.0007 T^{2}\right) \mathrm{fm} .
\end{gathered}
$$

This later formula proposed in Ref [16] allows to reproduce the small increase of the ratio $r_{0}=R_{0} / A^{1 / 3}$ with the mass ; for example, $r_{0}=1.11 \mathrm{fm}$ for ${ }^{20} \mathrm{Ne}$ and $r_{0}=1.18 \mathrm{fm}$ for ${ }^{240} \mathrm{Pu}$.

For comparison, the set of parameters used in the original version of the liquid drop model [21] was : $a_{s}=17.9439 \mathrm{MeV}$ and $r_{0}=1.2249 \mathrm{fm}$. The potential defined by Krappe, Nix and Sierk [24] assumes $a_{s}=21.7 \mathrm{MeV}$ and $r_{0}=1.18 \mathrm{fm}$ while the recent version of the Thomas-Fermi model [25] supposes $a_{s}=18.63 \mathrm{MeV}$ and $r_{0}=1.14 \mathrm{fm}$.

The surface and volume asymmetry coefficients take on the values:

$$
k_{s}=2.6 \text { and } k_{v}=1.8 \text {. }
$$

When the two fragments (or the two colliding nuclei) are separated :

$$
\begin{aligned}
& E_{V}=-a_{v}\left[\left(1-k_{v} I_{1}^{2}\right) A_{1}+\left(1-k_{v} I_{2}^{2}\right) A_{2}\right], \\
& E_{S}=a_{s}\left[\left(1-k_{s} I_{1}^{2}\right) A_{1}^{2 / 3}+\left(1-k_{s} I_{2}^{2}\right) A_{2}^{2 / 3}\right], \\
& E_{c}=\frac{3}{5} e^{2} Z_{1}^{2} / R_{1}+\frac{3}{5} e^{2} Z_{2}^{2} / R_{2}+e^{2} Z_{1} Z_{2} / r
\end{aligned}
$$

where $A_{i}, Z_{i}, R_{i}$ and $I_{i}$ are the masses, charges, radii and relative neutron excesses of the fragments and $r$ the distance between the mass centres.

The discontinuity of a few $\mathrm{MeV}$ which appears at the contact point when $\mathrm{Z}_{1} / \mathrm{A}_{1}$ and $Z_{2} / A_{2}$ are very different has been removed linearly from the contact point to the sphere since it is due to the progressive rearrangement of the nuclear matter.

The surface energy $E_{S}$ takes only into account the effects of the surface tension forces in an half space and does not include the contribution due to the attractive nuclear forces between the surfaces in regard in the neck or the gap between the nascent fragments. The nuclear proximity energy term $E_{N}$ allows to take into account these additional surface effects when crevices appear in the deformation path $[16,17,19]$.

$$
E_{N}=2 \gamma \int_{h_{\min }}^{h_{\max }} \phi(D / b) 2 \pi h d h .
$$

$h$ is the ring radius in the plane perpendicular to the fission axis and D the distance between the infinitesimal surfaces (see Fig. 1).

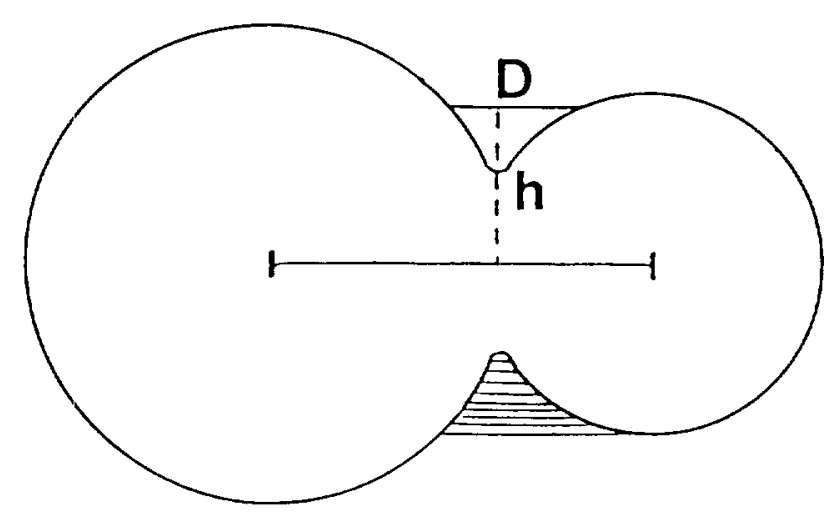

Fig. 1. Asrea of the proximity force influence and definition of $\mathrm{D}$ and $\mathrm{h}$. 
$b$ is the surface width fixed at the standard value of $0.99 \mathrm{fm}$. The $\phi$ function is taken with the parametrisation of Feldmeier [17]. The surface parameter $\gamma$ is given by a geometric mean between the surface parameters of the two fragments :

$$
\gamma=0.9517 \sqrt{\left(1-k_{s} I_{1}^{2}\right)\left(1-k_{s} I_{2}^{2}\right)} M e V . f m^{-2} \text {. }
$$

In this generalized liquid drop model the surface diffuseness is not considered in the surface energy term and the proximity energy vanishes when there is no neck as for ellipsoids for example.

The rotational energy has been determined within the rigid body ansatz. Indeed, it has been shown that corrective terms arising from the orbital motion and the spin degrees of freedom roughly cancel each other, particularly at large deformations.

$$
E_{\text {Rot }}=\frac{\hbar^{2} l(l+1)}{2 I_{\perp}} \text {. }
$$

\section{Quasi-molecular shapes}

A two parameter shape sequence has been defined $[19,22]$ to describe the continuous transition from one initial spherical nucleus to two tangent spherical fragments (see Fig. 2).

$$
R(\theta)^{2}=\left\{\begin{array}{ll}
a^{2} \sin ^{2} \theta+c_{1}^{2} \cos ^{2} \theta & (0 \leq \theta \leq \pi / 2) \\
a^{2} \sin ^{2} \theta+c_{2}^{2} \cos ^{2} \theta & (\pi / 2 \leq \theta \leq \pi)
\end{array} .\right.
$$

$c_{1}$ and $c_{2}$ are the two radial elongations and a the neck radius. This family of shapes is derived from the elliptic lemniscatoids obtained by inversion of axially symmetric ellipsoids [26]. Assuming volume conservation, the two parameters $s_{1}=a / c_{1}$ and $s_{2}=a / c_{2}$ completely define the shape. For a given decay channel, the ratio $\eta=R_{2} / R_{1}$ between the radii of the future fragments allows to connect $s_{1}$ and $s_{2}$ :

$$
s_{2}^{2}=\frac{s_{1}^{2}}{s_{1}^{2}+\left(1-s_{1}^{2}\right) \eta^{2}} . \quad\left(0 \leq s_{1}, s_{2}, \eta \leq 1\right)
$$
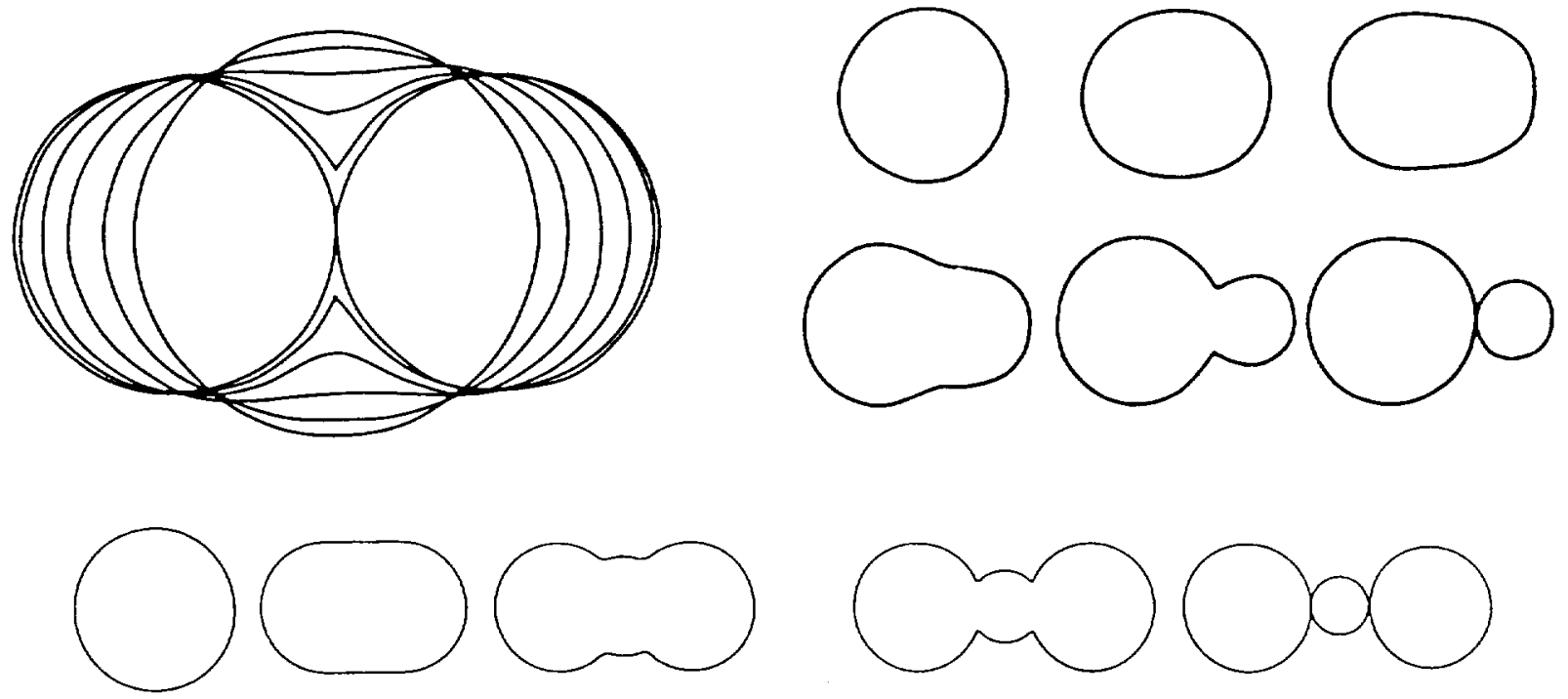

Fig. 2. Symmetric and asymmetric shapes leading to binary quasi-molecular configurations and generalization to ternary lission. 
When $s_{1}$ decreases from 1 to 0 the shape evolves continously from one sphere to two touching spheres with the natural formation of a deep neck while keeping almost spherical ends.

Using the axial symmetry, analytical expressions have been obtained for the various shape-dependent functions : volume, surface, moment of inertia, distance between the mass centres of each fragment and quadrupole moment [19].

\section{Symmetric fission barriers}

Assuming volume conservation, the volume energy is constant during the symmetric fission process. The different varying contributions to the potential energy are given separately together with the deformation energy of the ${ }^{160}$ Dy nucleus in Fig. 3 . The slope of the $E_{s}$ and $E_{N}$ curves changes drastically at the contact point since the surface is constant after the separation of the two spherical fragments and since the nuclear attraction is greatest at the contact point. Nevertheless, the total energy varies gently even around the contact point.

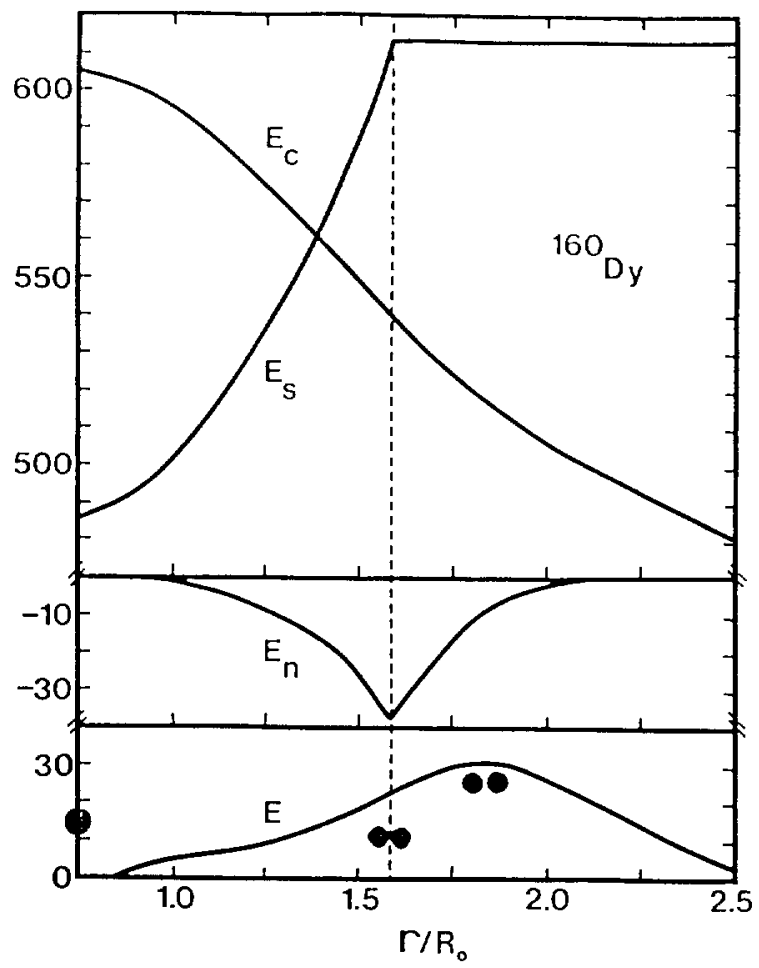

Fig. 3. Variation of the surface energy $E_{s}$, the Coulomb energy $E_{c}$, the nuclear proximity energy $E_{N}$ and the deformation energy $\mathrm{E}$ (in $\mathrm{MeV}$ ) for the ${ }^{160} \mathrm{Dy}$ nucleus plotted against the reduced distance between the mass centres in the fusion-like fission valley. The dashed line corresponds to the separation into two equal spherical fragments.

The deformation energies of the ${ }^{234} U$ nucleus corresponding to different shape sequences with and without proximity energy contribution are compared in Fig. 4. The dotted curve is the potential energy calculated using quasi-molecular shapes without taking into account the finite range effects of the nuclear forces. This shape sequence is then very energetically unfavourable. The thick full curve includes the proximity energy, on the same shape sequence, and may be compared with the thin full curve representing the deformation energy of the liquid drop model without proximity for usual elongated and little or not creviced shapes [10] 
This figure shows clearly that the comparison between the two shape sequences must be re-examined when the additional proximity energy term is introduced [22]. Nix and Swiatecki [27] using a two-spheroid model and no proximity contribution were already able to reproduce correctly the most probable values and widths of the experimental fragment mass distributions as well as the total kinetic energy for nuclei lighter than radium. The fission barrier heights were a few $\mathrm{MeV}$ too high and this model was considered as a rough approximation. Most of the subsequent studies [28] have used the development of the nuclear radius in terms of Legendre polynomials which, implicitly, is unable to generate shapes with deep necks. However, if the proximity energy is introduced, the difference between the two spheroid model (which is a generalisation of the two separated sphere model) and the more general parametrisations for one-body shapes is reversed because, for the two-spheroid model, the proximity energy is large due to the deep gap while this energy is negligible for the usual elongated one-body shapes. This can be seen in Fig. 4 where the chain curve (taken from [11]) gives the energy of two separated oblate spheroids (the proximity energy being not introduced). For $r / R_{0}<2$, this shape is soon favourable and this shape would be highly competitive with regard to the usual elongated shapes if the proximity energy was included.

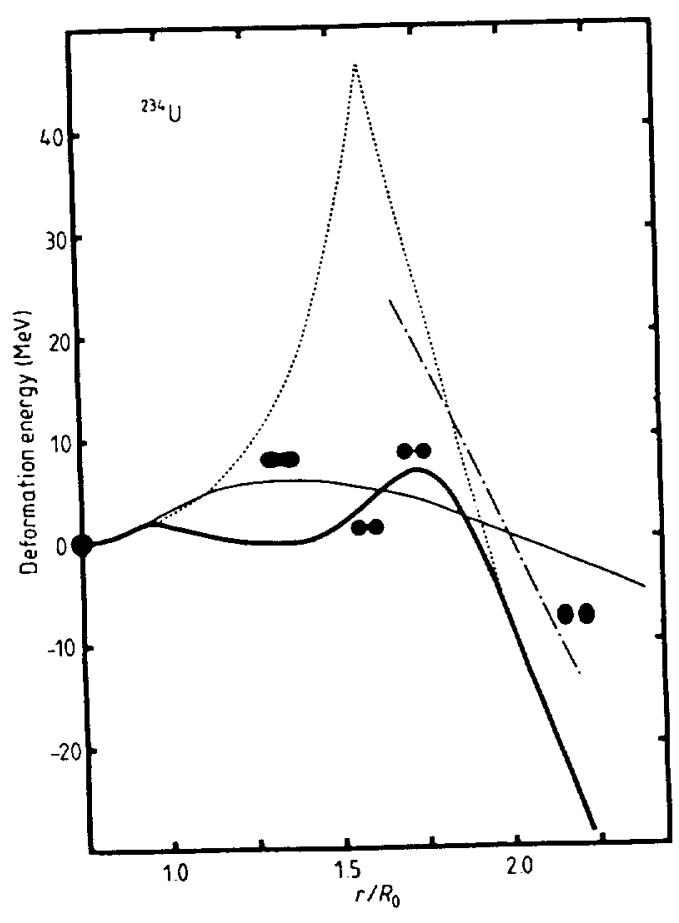

Fig. 4. Deformation energies of the ${ }^{234} U$ nucleus as a function of the relative distance between mass centres $r / R_{0}$. The thin full curve is the energy given by the original liquid drop model and for elongated shapes with shallow necks. The chain curve gives the energy of two separated oblate spheroids [11] with no proximity contribution. The thick full curve and the dotted curve are respectively the potential energies using our shape sequence with and without proximity energy.

The macroscopic fission barriers of five nuclei are plotted in Fig. 5. The proximity energy progressively introduces an inflection in the curve. for $\mathrm{A}<215$ there is one saddle point corresponding to two separated spherical fragments maintained in unstable equilibrium by the balance between the repulsive Coulomb forces and the attractive nuclear proximity forces. For $\mathrm{A}>215$, there are two maxima. The inner is close to the sphere while the outer always has the same configuration; between them a second minimum occurs due to the proximity effects in the neck which strongly lowers the 
deformation energy. The main characteristics of these barriers are the permanence of an outer peak which slows down the fragment separation and the appearance of macroscopic double-humped barriers for actinides. Nörenberg [29,30] has called any maximum in the potential energy which occurs after the fragment separation a scission barrier. For light-medium nuclei, the introduction of the proximity forces on such a shape sequence leads to the identification of fission and scission barriers, since the rupture of the neck between the fragments is assumed before the barrier is crossed. For heavier nuclei, double humped barriers have effectively been observed experimentally. The understanding of the nature of these complex structures needs to go beyond the mere minimisation of a liquid drop model energy with respect to deformations. Two main improvements have been widely advocated. Strutinsky [31] found double-humped barriers when taking into account shell and pairing corrections on elongated scission configurations without any proximity contribution. Nörenberg et al $[29,30,32]$ and Wilkins et al [33] restricting themselves to the interaction of two fragments have shown also the occurrence of an outer barrier when introducing the proximity interaction and the shell and pairing corrections; this scission barrier allowing to explain the angular momentum distribution of fission fragments. Our study points out the possibility to identify the scission barrier of Nörenberg et al with the outer peak of the double-humped barrier appearing in the actinide region.

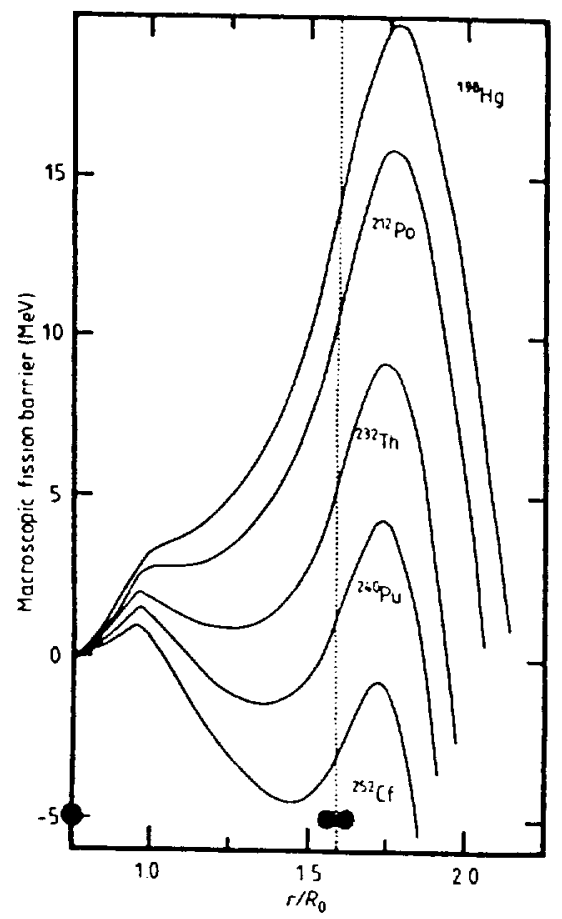

Fig. 5. Macroscopic fission barriers for ${ }^{198} \mathrm{Hg},{ }^{212} \mathrm{Po},{ }^{232} \mathrm{Th},{ }^{240} \mathrm{Pu}$ and ${ }^{252} \mathrm{Cf}$ as a function of the relative distance between mass centres. The dotted line corresponds to the contact point between the nascent spherical fragments.

The macroscopic fission barrier heights are displayed in Fig. 6. For the heaviest nuclei, the fission barrier height corresponds to the height of the second peak relatively to the second well minimum. The fission barrier heights in the deformation valley through quasi-molecular shapes agree remarkably well with those obtained by Krappe et al [24]; the differences in the macroscopic energy definition being compensated with the differences between the shape parametrisations. When compared with the pure liquid-drop estimations, which are recognised experimentally as being overestimated for the medium systems, the proximity energy strongly lowers the barrier height (typically $10 \mathrm{MeV}$ for $\mathrm{A}=100$ ). 


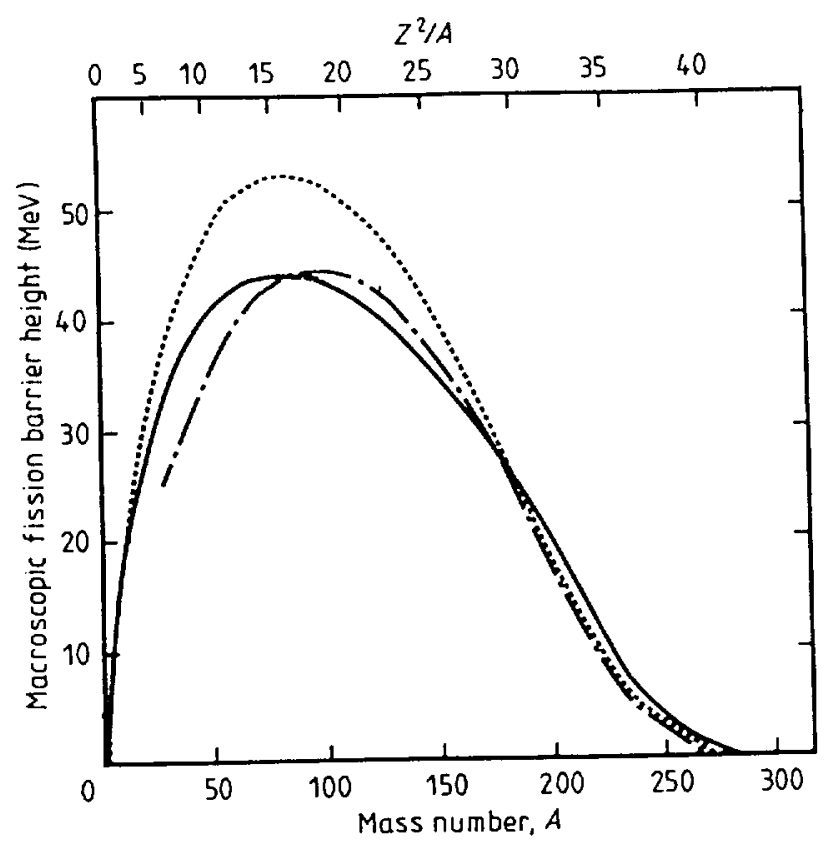

Fig. 6. Comparison of macroscopic fission barrier heights of $\beta$-stable nuclei. The dotted curve and the chain curve give respectively the predictions of the Liquid Drop Model [21] and of the Yukawa plus exponential Liquid Drop Model [24]. Our results within compact and necked shapes are shown by the full curve.

The shell corrections have been introduced as defined in the Droplet Model [34] with an attenuation factor given by

$$
E_{\text {Shell }}=E_{\text {Shell }}^{\text {sphere }}\left(1-2 \alpha^{2}\right) \exp \left(-\alpha^{2}\right) \quad \text { where } \quad \alpha^{2}=(\delta R)^{2} / a^{2} .
$$

The distortion $\alpha a$ is the root mean square of the deviation of the surface from a sphere, a quantity which incorporates all types of deformation indiscriminately. The range $a$ has been chosen to be $0.32 r_{0}$. Using this approach, shell corrections only play a role near the ground state of the compound nucleus and not at the saddle-point (where they are expected to be of the order of $1 \mathrm{MeV}$ or smaller). It has been clearly demonstrated within a single-particle model with pairing correlations [29,30] that, for two separated spheroids, the shell effects are strongly diminished since they are properties of valence nucleons and that the orbitals of which are strongly perturbed by the nuclear proximity potential. Thus, as soon as the shape is creviced, the application of the standard shell corrections to the liquid drop model energy seems to overestimate the veritable shell effects which are, really, partially destroyed by the proximity forces.

In Table 1, the macroscopic-microscopic fission barrier heights obtained in the quasi-molecular deformation valley within our generalized liquid drop model are compared with the results of the Droplet Model [34] and the experimental data. The agreement is fair in the whole range of nuclei considered. For the intermediate mass nuclei, this version of the Droplet Model gives too high barriers.

The double-humped barrier obtained for ${ }^{240} \mathrm{Pu}$ within the present generalized liquid drop model and the compact and creviced shapes is compared in Fig. 7 with the barrier determined by Möller and Nix [38]. The first minimum is quite similar in the two studies. The second barriers have the same height but do not lie at the same position. Experimentally, the quadrupole moment of the second minimum is around $36 \mathrm{~b}$. We obtain $46.7 \mathrm{~b}$ but the well is very flat. A deformation leading to $36 \mathrm{~b}$ lies only $0.5 \mathrm{MeV}$ above the calculated minimum.

The main result is the purely macroscopic origin of the second barrier and well for the quasi-molecular shapes. In contrast, for elongated shapes, shell effects must be introduced to generate a second barrier at large deformations. 
Table 1. Comparison of experimental [35,36] and theoretical [22,34,37] fission barrier heights. For the actinide nuclei, only the outer barrier height is included.

\begin{tabular}{|c|c|c|c|c|c|c|c|}
\hline \multicolumn{7}{|c|}{ Symmetric fission barrier heights (MeV) } \\
\hline Nucleus & Exp. data & $\begin{array}{c}\text { Present } \\
\text { study }\end{array}$ & $\begin{array}{c}\text { Droplet } \\
\text { Model }\end{array}$ & Nucleus & Exp. data & $\begin{array}{c}\text { Present } \\
\text { study }\end{array}$ & $\begin{array}{c}\text { Droplet } \\
\text { model }\end{array}$ \\
\hline${ }^{109} \mathrm{Cd}$ & 34.0 & 40.8 & - & ${ }^{209} \mathrm{Bi}$ & $21.9-25.5$ & 25.0 & 22.4 \\
\hline${ }^{149} \mathrm{Eu}$ & $32.5 \pm 2$ & 33.7 & 40.4 & ${ }^{212} \mathrm{Po}$ & 19.7 & 21.4 & - \\
\hline${ }^{152} \mathrm{~Tb}$ & $29.0 \pm 1$ & 32.0 & 37.1 & ${ }^{213} \mathrm{At}$ & $14.3-17.2$ & 19.5 & 16.9 \\
\hline${ }^{157} \mathrm{Ho}$ & $26.5 \pm 2$ & 31.3 & 34.5 & ${ }^{216} \mathrm{Rn}$ & $13.1 \pm 1.0$ & 16.1 & 13.5 \\
\hline${ }^{160} \mathrm{Dy}$ & 27.4 & 33.9 & - & ${ }^{226} \mathrm{Ac}$ & 8.0 & 9.0 & 5.3 \\
\hline${ }^{173} \mathrm{Lu}$ & $27.1-31.5$ & 30.0 & 30.4 & ${ }^{228} \mathrm{Ra}$ & 8.1 & 9.9 & - \\
\hline${ }^{179} \mathrm{Ta}$ & $25.4-29.2$ & 27.9 & 27.1 & ${ }^{232} \mathrm{Th}$ & 6.0 & 8.3 & - \\
\hline${ }^{180} \mathrm{~W}$ & $23.9-28.7$ & 26.7 & 25.6 & ${ }^{234} \mathrm{U}$ & 6.0 & 6.9 & \\
\hline${ }^{185} \mathrm{Re}$ & $24.0-26.4$ & 25.5 & 23.6 & ${ }^{238} \mathrm{U}$ & $5.1-5.8$ & 7.0 & 4.9 \\
\hline${ }^{186} \mathrm{Os}$ & 24.0 & 24.2 & - & ${ }^{240} \mathrm{Pu}$ & 5.7 & 5.7 & - \\
\hline${ }^{191} \mathrm{Ir}$ & $20.6-23.7$ & 22.7 & 21.9 & ${ }^{242} \mathrm{Am}$ & 6.4 & 5.2 & 4.4 \\
\hline${ }^{195} \mathrm{Au}$ & $18.6-21.6$ & 21.1 & 20.9 & ${ }^{244} \mathrm{Pu}$ & $5.4 \pm 0.3$ & 5.7 & 4.6 \\
\hline${ }^{198} \mathrm{Hg}$ & 21.8 & 21.9 & - & ${ }^{246} \mathrm{Cm}$ & 4.7 & 4.7 & - \\
\hline${ }^{201} \mathrm{Tl}$ & $19.5-23.5$ & 22.8 & 21.3 & ${ }^{250} \mathrm{Bk}$ & 5.8 & 4.2 & 4.1 \\
\hline${ }^{206} \mathrm{~Pb}$ & $26.0-26.8$ & 25.4 & 23.0 & ${ }^{233} \mathrm{Cf}$ & $5.3 \pm 0.3$ & 3.7 & 3.9 \\
\hline
\end{tabular}

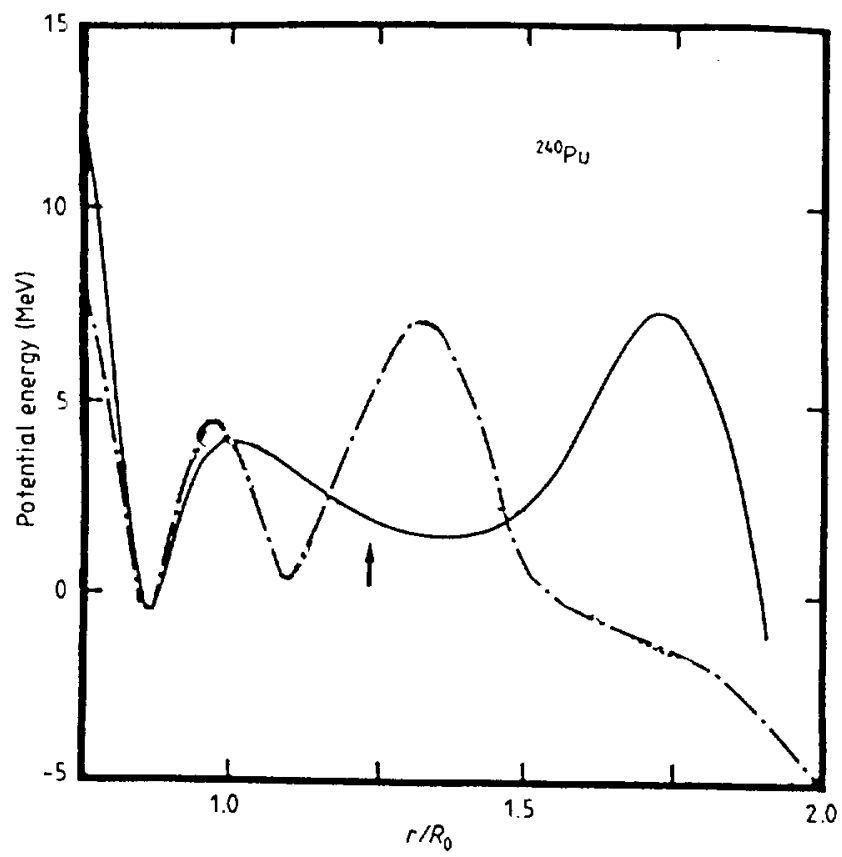

Fig. 7. Macroscopic-microscopic potential energy for ${ }^{240} \mathrm{Pu}$ as a function of $r / \mathrm{R}_{0}$. The double-humped potential barrier obtained in the quasi-molecular shape valley is given by the full curve [22]. The chain curve shows the barrier obtained using the folded Yukawa shell model potential and elongated shapes [38]. The arrow gives the experimental position of the second well.

The macroscopic-microscopic potential barriers existing for very heavy and superheavy elements in the fusion-like fission valley are displayed in Figs 8 and 9. The macroscopic contribution to the first peak diminishes continuously and vanishes for the heaviest nuclei ; their stability being therefore due uniquely to shell effects. The second peak progressively vanishes also for the heavy nuclei because the attractive nuclear force can no longer equilibrate the repulsive Coulomb force. The second peak disappears completely for the superheavy elements due to the dominant Coulomb repulsion. No 
isomeric state can exist for these systems and they only survive in their ground state. If such a barrier reproduces the physical reality, the hope of forming these superheavy elements in fusion reactions is strongly reduced. The slope of the potential barrier to pass for reaching the almost spherical compound nucleus is very pronounced and the barriers are thinner than those found in other studies.

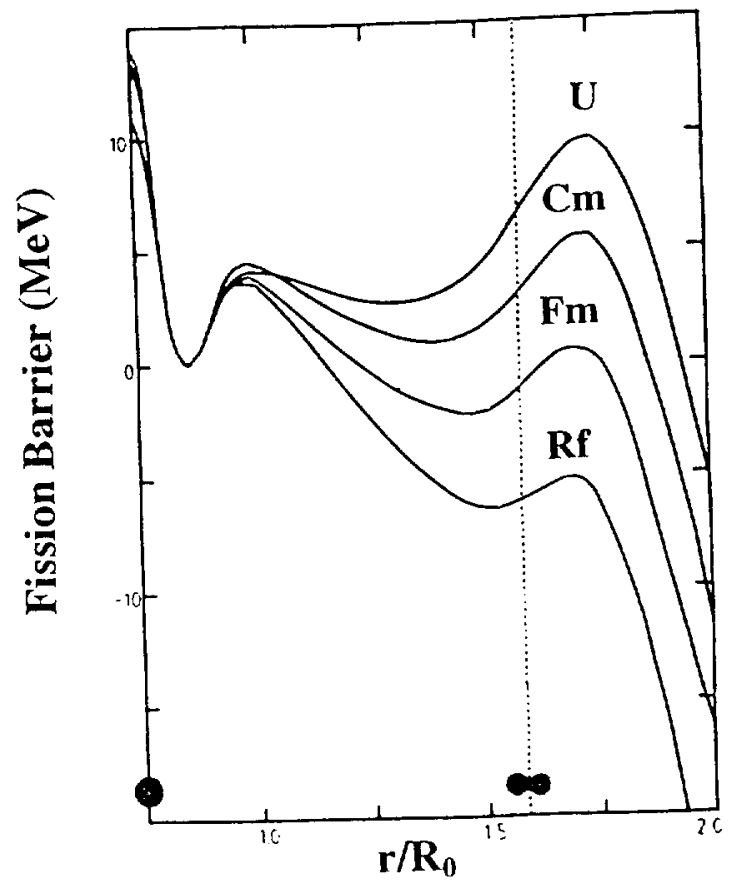

Fig. 8. Macroscopic-microscopic potential energy for ${ }^{238} \mathrm{U},{ }^{246} \mathrm{Cm},{ }^{258} \mathrm{Fm}$ and ${ }^{260} \mathrm{Rf}$ as a function of $\mathrm{r} / \mathrm{R}_{0}$.

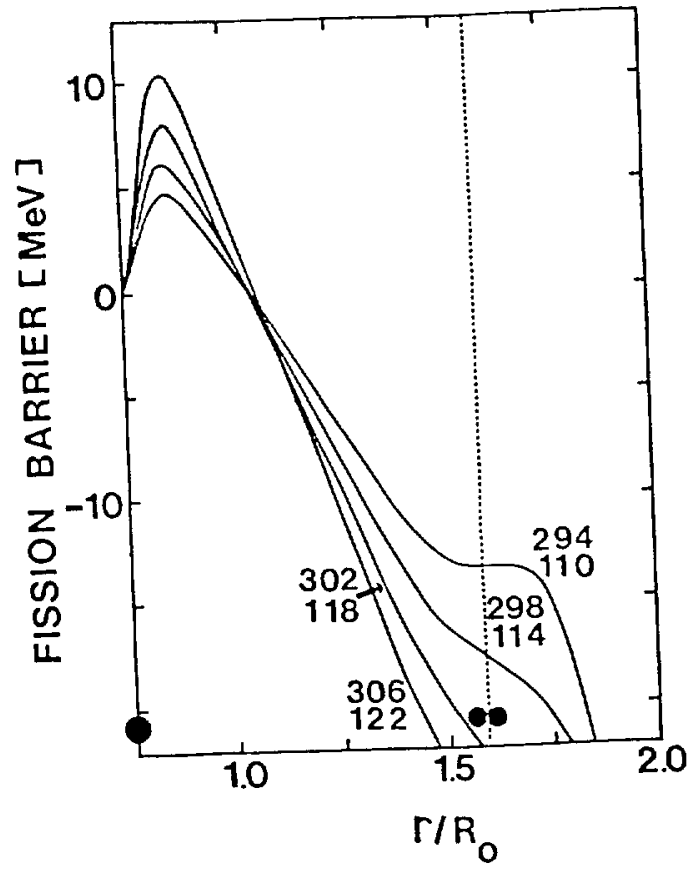

Fig. 9. Macroscopic-microscopic potential energy of the superheavy nuclei ${ }_{110}^{294} \mathrm{Sh},{ }_{114}^{298} \mathrm{Sh},{ }_{118}^{302} \mathrm{Sh}$ and ${ }_{122}^{306} \mathrm{Sh}$.

Different characteristics of the double-humped barriers for actinides are summarised in Table 2 . The second barrier heights are systematically too high for the light actinides but the same difficulty has been encountered in other studies before assuming asymmetry. Nevertheless, these results remain inside the precision that we may expect from a one parameter shape sequence and without adjustment of the liquid drop model coefficients. 
Table 2. Comparison between calculated and experimental barrier characteristics of six actinide nuclei. The exper mental data are taken from [39]. $v_{a}, v_{b}$ and $E_{I I}$ are respectively the first peak height, the second peak height and the energy of the second potential minimum relatively to the ground state (in $\mathrm{MeV}$ ).

\begin{tabular}{|c|c|c|c|c|c|c|}
\hline Isotope & $\mathrm{v}_{\mathrm{a}}$ expt & $\mathrm{v}_{\mathrm{a}}$ theory & $\mathrm{E}_{\mathrm{II}}$ expt & $\mathrm{E}_{\mathrm{II}}$ theory & $\mathrm{v}_{\mathrm{b}}$ expt & $\mathrm{V}_{\mathrm{b}}$ theory \\
\hline${ }^{232} \mathrm{Th}$ & 5.8 & 3.7 & $<4.5$ & 2.9 & 6.2 & 11.2 \\
\hline${ }^{234} \mathrm{Th}$ & 6.1 & 3.9 & & 3.1 & 6.5 & 11.5 \\
\hline${ }^{234} \mathrm{U}$ & 5.6 & 3.8 & & 2.2 & 5.5 & 9.1 \\
\hline${ }^{238} \mathrm{U}$ & 5.7 & 4.2 & 2.6 & 2.7 & 5.7 & 9.7 \\
\hline${ }^{240} \mathrm{Pu}$ & 5.6 & 4.3 & 2.4 & 1.7 & 5.1 & 7.4 \\
\hline${ }^{246} \mathrm{Cm}$ & 5.7 & 4.5 & & 0.8 & 4.2 & 5.5 \\
\hline
\end{tabular}

\section{Asymmetric fission barriers}

In this section, the compatibility of an asymmetric decay process through these quasi-molecular shapes [40] is checked with experimental data . In Fig. 10 calculations on the asymmetric breakup of ${ }^{149} \mathrm{~Tb}$ are compared with recent results. It is known that the original version of the LDM [28] predicts too high fission barrier heights for light and intermediate mass nuclei. Here, the difference with the data is only $10 \%$. In contrast, the experimental barriers are 37\% greater than those determined by the YEFRLDM [24] which, nevertheless, reproduces generally accurately nuclear masses and fission and fusion barrier heights. Our calculations agree well with the data. Fig 11 shows that our approach reproduces also fairly well the recent data for ${ }^{94} \mathrm{Mo}$ (except for the lighest fragments) while it overestimates slightly the barriers for the In nucleus. In all cases, our asymmetric fission barrier heights lie between the predictions of the LDM and YEFRLDM models.

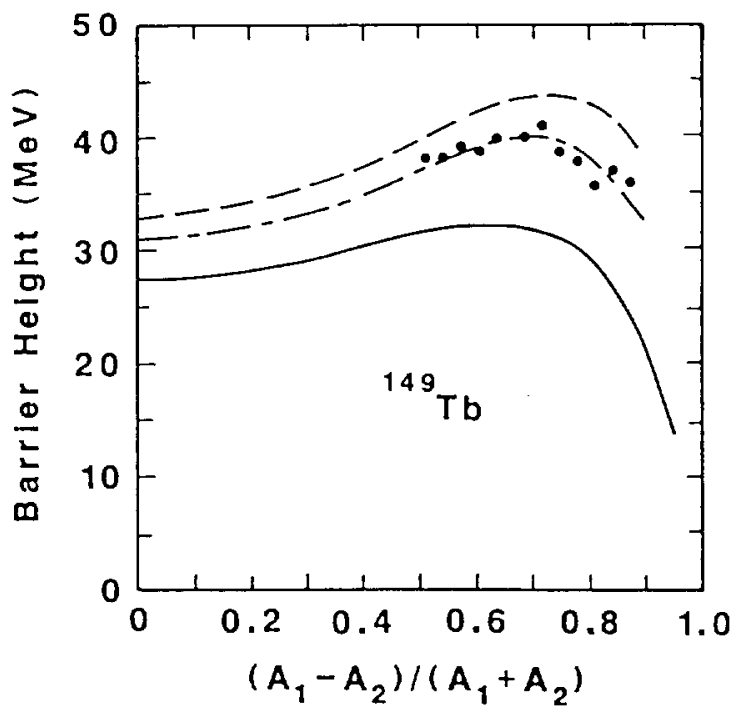

Fig. 10. Comparison of experimental data [6] and theoretical fission barrier heights for ${ }^{149} \mathrm{~Tb}$ versus the fission asymmetry. The solid and dashed lines correspond respectively to the YEFRLDM [24,42] and LDM [28] while our results are given by the dashed-dotted curve [40].

It is also important to know whether, in this fusion-like fission valley, the Businaro-Gallone point [43] where nuclei become unstable with regard to asymmetric breakup is also correctly localized in the nuclear mass range. The potential surfaces seen by the fissionning ${ }^{86} \mathrm{Kr}$ and ${ }^{205} \mathrm{At}$ nuclei are displayed in Fig. 12. As expected, for the light nucleus the barrier is maximal for symmetric fission and decreases monotically 
with asymmetry. Thus, the potential favours decay by light fragment emission. On the other hand, the heavy nucleus shows the opposite behaviour. The symmetric fission path corresponds to the exit channel which minimizes the potential energy. The barrier heights reach a maximum for a very asymmetric configuration. For still greater asymmetry, the barrier decreases again. For this intermediate mass range, decays by symmetric fission and light charged particle emission are favoured. For actinide nuclei, the macroscopic fission barrier heights (around $5 \mathrm{MeV}$ ) are of the same order as the shell effects and, consequently, the dependence on the mass asymmetry is not smooth. Furthermore, for the heaviest elements, the decay via alpha particle emission is the most important one.
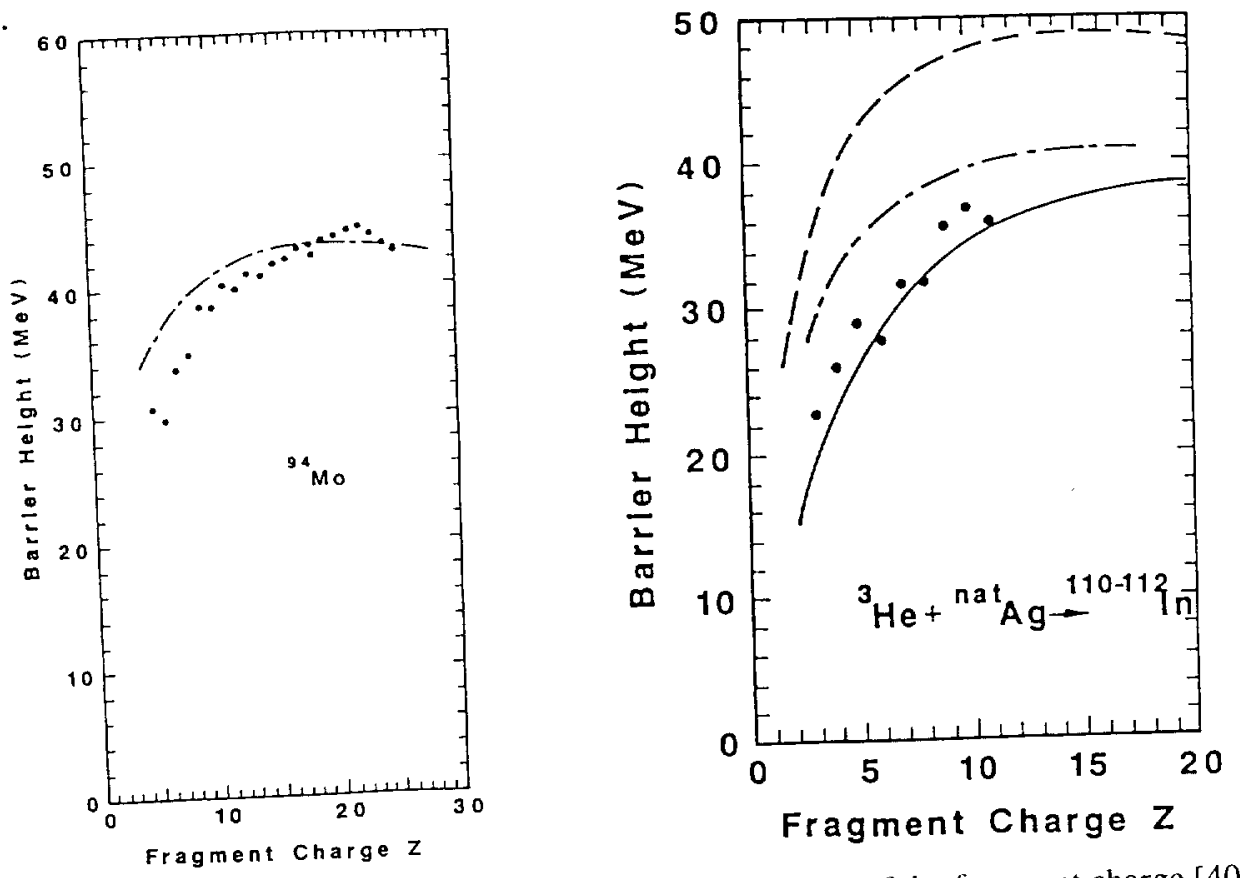

Fig. 11. Same as Fig. 10 but for ${ }^{94} \mathrm{Mo}$ and ${ }^{110-112}$ In and as a function of the fragment charge [40].

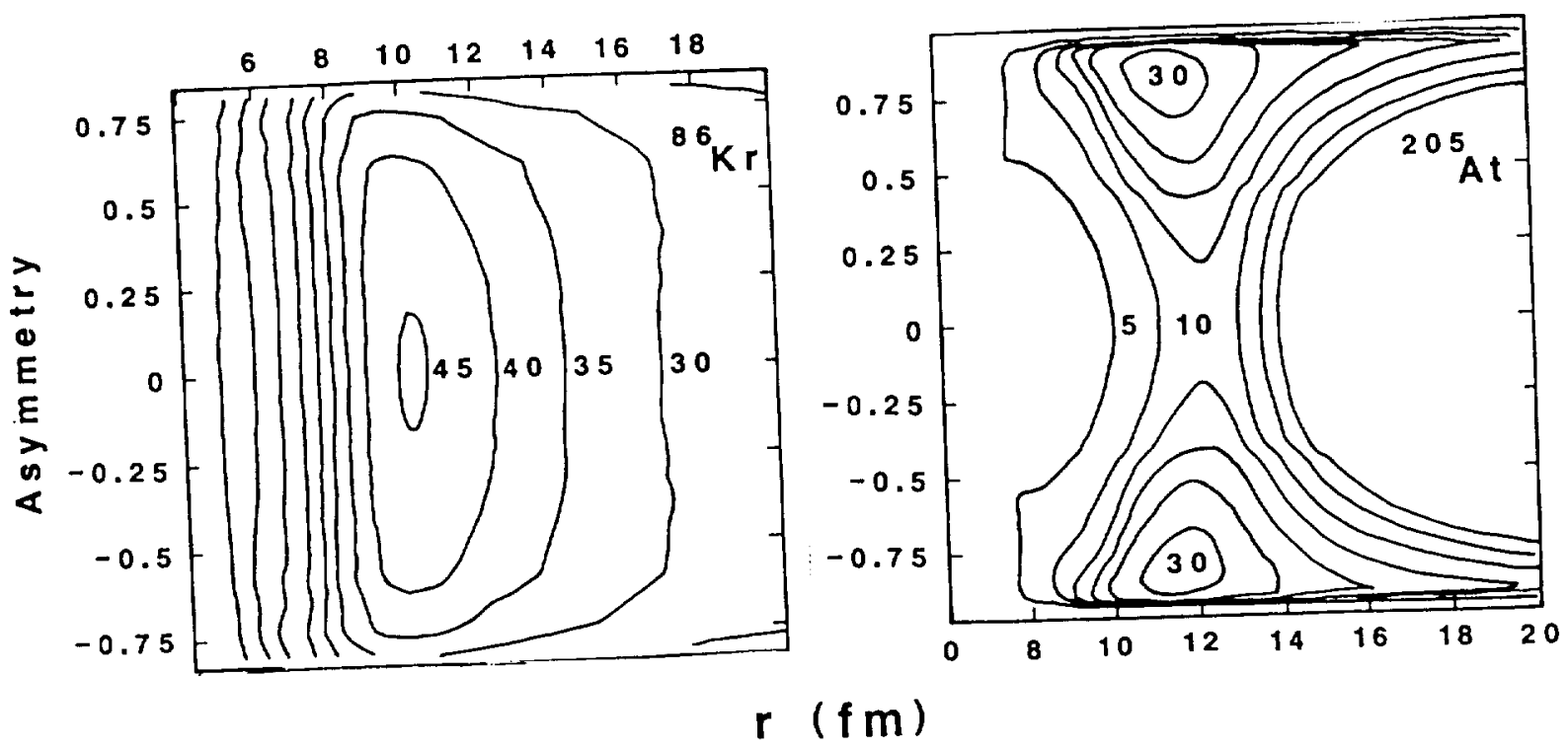

Fig. 12. Potential energy surface versus the mass asymmetry $\left(A_{1}-A_{2}\right) / A$ and the distance between the mass centres for typical light and heavy nuclei. 
The so-called 'conditional' saddle-point (CSP) corresponds to the barrier for a fixed mass asymmetry. When all these CSP are plotted in a single curve, they form a ridge line summarising the main information (see Fig. 13). The often used fissility coefficient $\mathrm{x}=\mathrm{E}_{\mathrm{Coul}} / 2 \mathrm{E}_{\text {surf }}$ gives the interplay between the Coulomb and the surface energies. It can lead to some confusion since the different macroscopic models do not use the same surface coefficients and the selected nucleus should always be specified. For light nuclei, the curve is monotonic and reaches its minimal value for very asymmetric splitting. As the nucleus becomes bigger, the symmetric configuration barriers decrease. For a fissility value between 0.4 and 0.5 the curve is a plateau and all the exit channels have very similar CSP values. For still heavier nuclei, the symmetric splitting turns out to have the lowest CSP. The ridge curve in this case reaches a maximum for very asymmetric configurations and then drops again for the most asymmetric decays. This is exactly the Businaro-Gallone picture. More precisely, the BG point falls in the mass interval 81-145 in our study in agreement with the experimental estimations which locate the BG point between 85-145 [44].

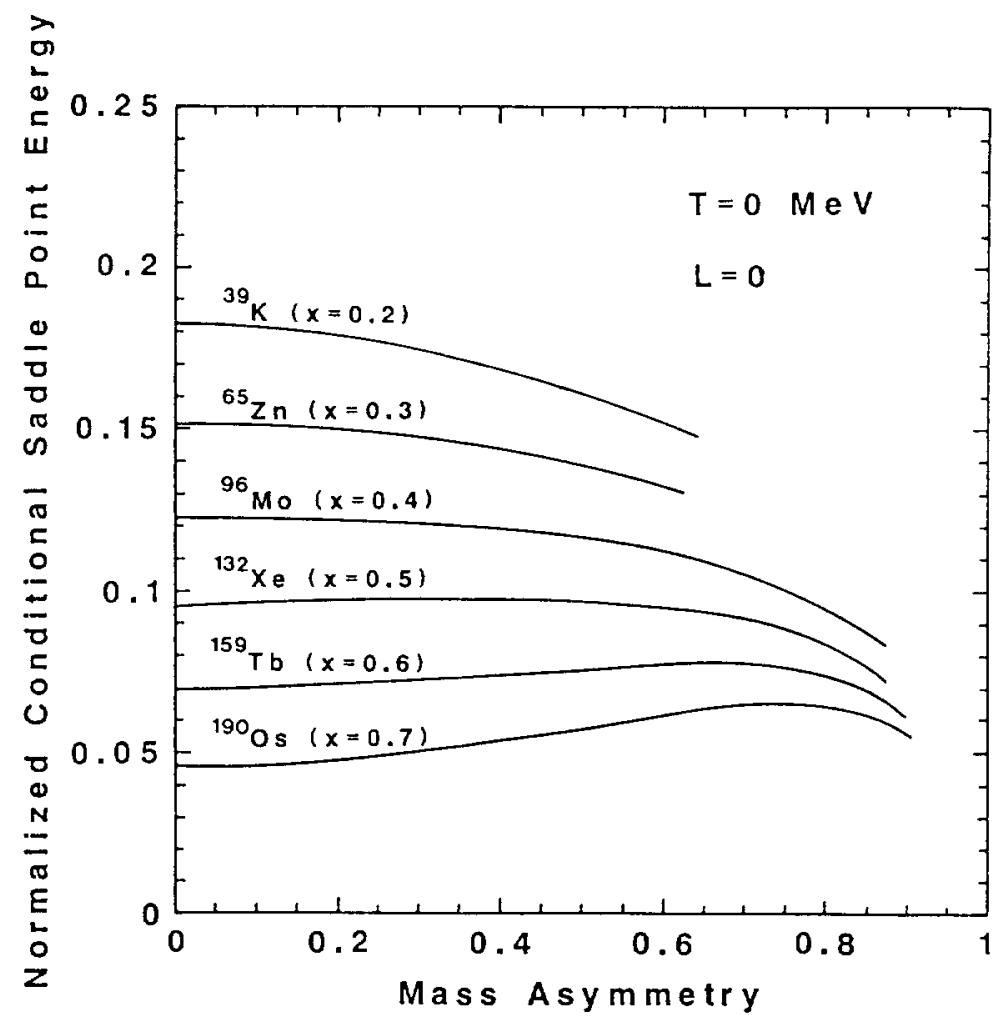

Fig. 13. Ridge lines for different $\beta$ stable nuclei. The conditional saddle-point energy is normalized to the surface energy of the corresponding compound nucleus.

\section{Emission of $\mathrm{C}, \mathrm{O}, \mathrm{Ne}, \mathrm{Mg}$ and $\mathrm{Si}$ nuclei}

The half-lives of nuclei emitting heavy clusters like $\mathrm{C}, \mathrm{O}, \mathrm{Ne}, \mathrm{Mg}$ and $\mathrm{Si}$ through this very asymmetric fission valley leading rapidly to two spherical touching nuclei at the early stage of the tunneling process have been calculated [45] and compared with recent data relative to the so-called cluster radioactivity $[3,4]$. In such an unified fission approach, the decay constant of the parent nucleus is simply $\lambda=v_{0} P$. There is no adjustable preformation factor. The assault frequency $v_{0}$ can be evaluated from the zero point vibration energy $E_{v}=1 / 2 h v_{0}$ and, in the harmonic oscillator approximation, 
$v_{0}=2.5 \times 10^{20} \mathrm{~s}^{-1}$. The barrier penetrability $P$ is calculated within the general form of the action integral

$$
P=\exp \left\{-\frac{2}{\hbar} \int_{R_{a}}^{R_{b}}\left[2 B(r)\left(E(r)-E\left(R_{a}\right)\right)\right]^{1 / 2} d r\right\},
$$

with $E\left(R_{a}\right)=E\left(R_{b}\right)=Q$.

The expression proposed for the inertia $B(r)$ in this new fusion-like fission valley [46] has been used

where $f(r)=\left\{\begin{array}{ll}\left(\frac{R_{\text {cont }}-r}{R_{\text {cont }}-R_{a}}\right)^{2} ; & r \leq R_{\text {cont }} \\ 0 ; & r \geq R_{\text {cont }}\end{array} \quad\right.$ and $\quad R_{\text {cont }}=R_{1}+R_{2}$.

The partial half-life time is related to the decay constant $\lambda$ by $T_{1 / 2}=\frac{\ln 2}{\lambda}$. No parameter used to determine the potential energy has been changed.

The predictions within the macroscopic LDM barrier tunneling and the experimental partial half-lives are compared in the table 3 (first and third columns). The macroscopic part of the total energy leads to a reasonable agreement for the heaviest clusters but fails to reproduce the ${ }^{20} \mathrm{O}$ and ${ }^{14} \mathrm{C}$ emission.

Table 3. Time characteristics (in s) of the cluster radioactivity. The first and second columns indicate respectively the theoretical half-lives without and with taking into account the microscopic corrections. The last column gives the experimental data.

\begin{tabular}{|c|c|c|c|}
\hline $\begin{array}{c}\text { Emitter } \\
\text { and } \\
\text { cluster }\end{array}$ & $\begin{array}{c}\text { Theoretical } T_{1 / 2}: \\
\text { macroscopic LDM } \\
\text { barrier tunneling }\end{array}$ & $\begin{array}{c}\text { Theoretical } T_{1 / 2}: \\
\text { macroscopic and } \\
\text { microscopic barrier } \\
\text { tunneling }\end{array}$ & $\begin{array}{c}\text { Experimental } \\
T_{1 / 2}(\mathrm{~s})\end{array}$ \\
\hline${ }^{222} \mathrm{Ra} \rightarrow{ }^{14} \mathrm{C}+{ }^{208} \mathrm{~Pb}$ & $2.7 \times 10^{33}$ & $2.0 \times 10^{11}$ & $1.2 \times 10^{11}$ \\
\hline${ }^{223} \mathrm{Ra} \rightarrow{ }^{14} \mathrm{C}+{ }^{209} \mathrm{~Pb}$ & $1.6 \times 10^{34}$ & $1.2 \times 10^{14}$ & $2.0 \times 10^{15}$ \\
\hline${ }^{224} \mathrm{Ra} \rightarrow{ }^{14} \mathrm{C}+{ }^{210} \mathrm{~Pb}$ & $1.1 \times 10^{35}$ & $1.9 \times 10^{17}$ & $7.4 \times 10^{15}$ \\
\hline${ }^{226} \mathrm{Ra} \rightarrow{ }^{14} \mathrm{C}+{ }^{212} \mathrm{~Pb}$ & $4.3 \times 10^{35}$ & $6.8 \times 10^{22}$ & $1.8 \times 10^{21}$ \\
\hline${ }^{228} \mathrm{Th} \rightarrow{ }^{20} \mathrm{O}+{ }^{208} \mathrm{~Pb}$ & $1.3 \times 10^{26}$ & $4.3 \times 10^{22}$ & $7.5 \times 10^{20}$ \\
\hline${ }^{230} \mathrm{Th} \rightarrow{ }^{24} \mathrm{Ne}+{ }^{206} \mathrm{Hg}$ & $1.1 \times 10^{26}$ & $3.7 \times 10^{26}$ & $4.4 \times 10^{24}$ \\
\hline${ }^{231} \mathrm{~Pa} \rightarrow{ }^{24} \mathrm{Ne}+{ }^{207} \mathrm{Tl}$ & $2.9 \times 10^{24}$ & $1.2 \times 10^{23}$ & $1.7 \times 10^{23}$ \\
\hline${ }^{232} \mathrm{U} \rightarrow{ }^{24} \mathrm{Ne}+{ }^{208} \mathrm{~Pb}$ & $9.6 \times 10^{22}$ & $1.3 \times 10^{21}$ & $2.5 \times 10^{20}$ \\
\hline${ }^{233} \mathrm{U} \rightarrow{ }^{24} \mathrm{Ne}+{ }^{209} \mathrm{~Pb}$ & $3.3 \times 10^{23}$ & $4.7 \times 10^{24}$ & $6.8 \times 10^{24}$ \\
\hline${ }^{234} \mathrm{U} \rightarrow{ }^{24} \mathrm{Ne}+{ }^{210} \mathrm{~Pb}$ & $1.2 \times 10^{24}$ & $9.4 \times 10^{27}$ & $1.6 \times 10^{25}$ \\
\hline${ }^{234} \mathrm{U} \rightarrow{ }^{28} \mathrm{Mg}+{ }^{206} \mathrm{Hg}$ & $7.6 \times 10^{24}$ & $1.4 \times 10^{27}$ & $3.5 \times 10^{25}$ \\
\hline${ }^{235} \mathrm{U} \rightarrow{ }^{28} \mathrm{Mg}+{ }^{207} \mathrm{Hg}$ & $5.1 \times 10^{25}$ & $4.6 \times 10^{30}$ & $2.8 \times 10^{28}$ \\
\hline${ }^{236} \mathrm{Pu} \rightarrow{ }^{28} \mathrm{Mg}+{ }^{208} \mathrm{~Pb}$ & $2.0 \times 10^{21}$ & $1.7 \times 10^{21}$ & $4.7 \times 10^{21}$ \\
\hline${ }^{238} \mathrm{Pu} \rightarrow{ }^{28} \mathrm{Mg}+{ }^{210} \mathrm{~Pb}$ & $6.2 \times 10^{22}$ & $8.0 \times 10^{27}$ & $5.0 \times 10^{25}$ \\
\hline${ }^{238} \mathrm{Pu} \rightarrow{ }^{32} \mathrm{Si}+{ }^{206} \mathrm{Hg}$ & $1.3 \times 10^{25}$ & $8.4 \times 10^{27}$ & $1.9 \times 10^{25}$ \\
\hline
\end{tabular}


In order to simulate the microscopic corrections such as shell effects and pairing in this very asymmetric decay case, the difference between the experimental Q-value and the theoretical one deduced from the present LDM has been added at the macroscopic potential energy of the initial spherical nucleus with a linear attenuation factor vanishing at the contact point of the nascent fragments. The theoretical estimates agree very well with the data for all the $\mathrm{C}, \mathrm{O}, \mathrm{Ne}, \mathrm{Mg}$ and $\mathrm{Si}$ clusters when the microscopic contributions are included (second column in the table).

Therefore, it seems that the emission of clusters by heavy nuclei may be viewed as the limiting case of very asymmetric fission via compact and creviced shapes. It is important to emphasize that the main explanation of the good reproduction of the experimental data is the ability of the present model to reproduce the height and width of the potential barriers with the help of the experimental Q-value. The physical process which leads to two tangent spherical fragments may be an adiabatic fission process or, alternatively, the emission of a preformed cluster. Whatever the physical process is, the role of the proximity energy and the microscopic corrections is emphasized since their introduction is sufficient to reproduce the potential barrier characteristics which govern the half-lives of the compound nuclei.

\section{Fragment kinetic energy}

The fragment kinetic energy is the potential energy at the scission point plus the possible pre-scission kinetic energy. The total energy released from saddle point to infinity is the sum of that kinetic energy and of the fragment excitation energy due to dissipation and deformation. The question then arises of the definition of the scission point. In the usual fission valley, the breaking of the matter bridge occurs for shallow necks between long touching fragments [47]. Then, the nuclear proximity forces are very weak and these rupture points between the fragments are chosen as scission points. Such a definition is impossible for quasi-molecular shapes since at the contact point between the spherical nascent fragments the proximity energy reaches $30-40 \mathrm{MeV}$. Nörenberg $[29,30]$ has defined the scission point as the top of the scission barrier where the deformations are small since the repulsive Coulomb forces are approximately cancelled by the attractive proximity forces. For ${ }^{240} \mathrm{Pu}$, the proximity energy is still $-18 \mathrm{MeV}$; the distance between the equivalent sharp surfaces being $1.1 \mathrm{fm}$. Wilkins et al [33] have arbitrarily fixed the scission point as the point where the distance between the surfaces is $1.4 \mathrm{fm}$ for all nuclei. For $A>100$ and along the $\beta$ stability valley, we have found that the distance between the equivalent sharp surfaces at the saddle point of the fission barrier is given by $-0.00647 \mathrm{~A}+2.587$.

The range of the nuclear friction is strongly correlated with the range of the nuclear force and the dissipation vanishes when the nuclear force effects disappear. Thus, the scission point where the fragments become free is more outer and lower than the saddle point. It is difficult to define its position precisely because it is located in the tail of the proximity energy and where the slope of the deformation energy is very pronounced. In Figs. 14 and 15, the Coulomb energy at the point where $\left|E_{N}\right|<0.5 \mathrm{MeV}$ is compared with the kinetic energy of the intermediate mass fragments produced during the asymmetric fission of ${ }^{149} \mathrm{~Tb}$ and ${ }^{194} \mathrm{Hg}$. The similarity between these two energies confirms that the definition of the scission point as the point where the proximity energy vanishes is reasonable. 


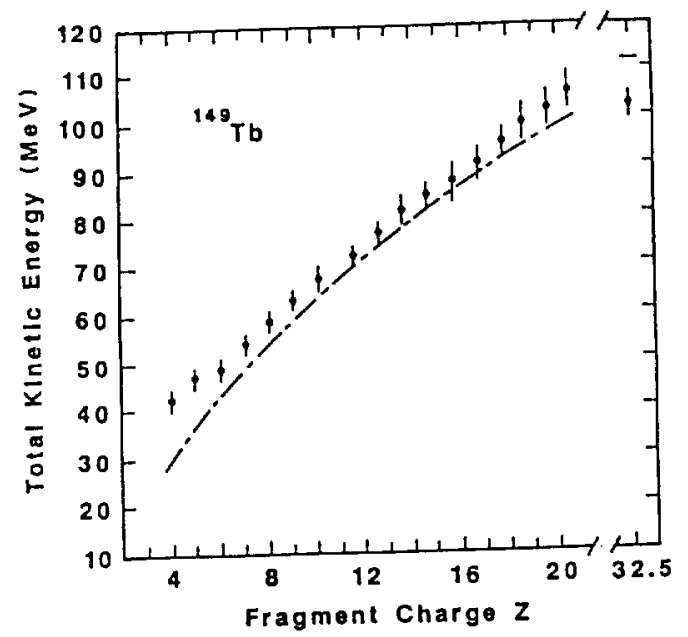

Fig. 14. Total kinetic energies as a function of the light fragment charge for ${ }^{149} \mathrm{~Tb}$ [40]. The theoretical estimate (dashed and dotted curve) is based upon the interaction Coulomb energy at the scission point defined by an absolute value of the proximity energy lower than $0.5 \mathrm{MeV}$. The experimental data are extracted from [48].

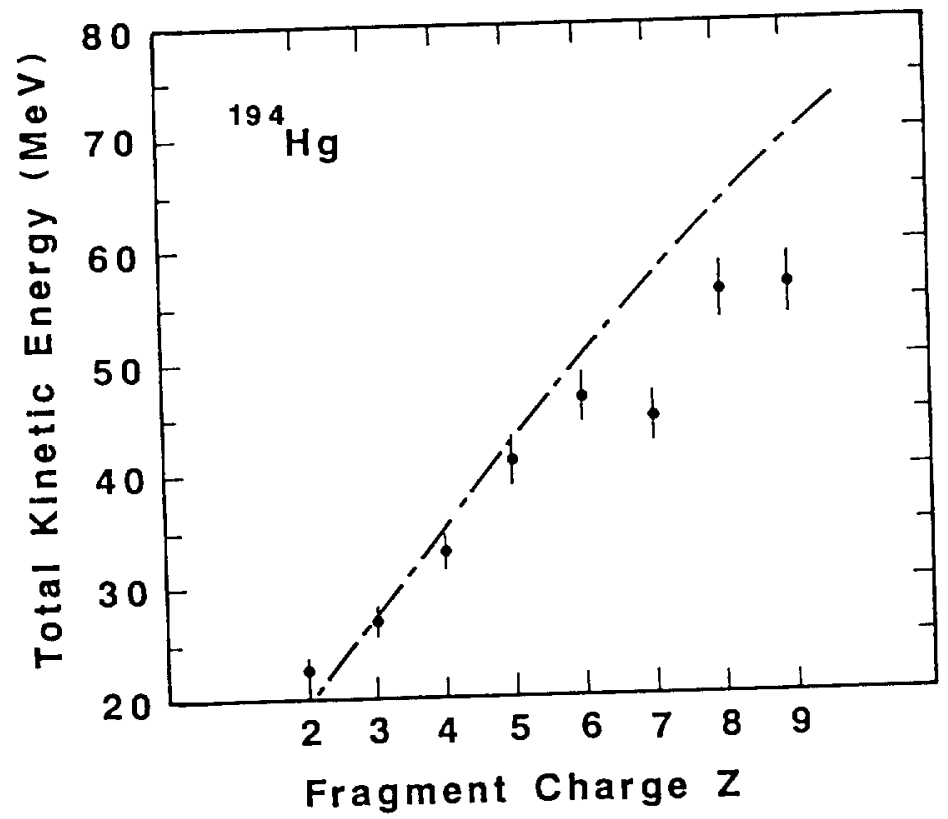

Fig. 15. Comparison between experimental TKE's [49] and theoretical predictions [40] for light fragment emission from ${ }^{194} \mathrm{Hg}$.

In table 4 , the dependence of the predicted total kinetic energy on the selected threshold value for the nuclear proximity energy is displayed and the predictions are compared with experimental data.

Table 4. Comparison of the experimental fragment kinetic energies estimated from [47] and theoretical total kinetic energies taken as the Coulomb interaction energies when the nuclear proximity energies are lower than $0.1,0.2$ and $0.5 \mathrm{MeV}$ in the case of symmetric fission.

\begin{tabular}{|c|c|c|c|c|c|}
\hline Nucleus & $\mathrm{Z}^{2} / \mathrm{A}^{1 / 3}$ & $\begin{array}{c}\text { Empirical TKE } \\
(\mathrm{MeV})\end{array}$ & $\begin{array}{c}\text { TKE } \\
\left|E_{N}\right|<0.1 \mathrm{MeV}\end{array}$ & $\begin{array}{c}\text { TKE } \\
\left|E_{N}\right|<0.2 \mathrm{MeV}\end{array}$ & $\left|E_{N}\right|<0.5 \mathrm{MeV}$ \\
\hline${ }^{257} \mathrm{Fm}$ & 1573 & 193 & 214 & 220 & 229 \\
\hline${ }^{226} \mathrm{Ra}$ & 1271 & 158 & 171 & 177 & 184 \\
\hline${ }^{192} \mathrm{Ir}$ & 1027 & 133 & 137 & 141 & 147 \\
\hline${ }^{162} \mathrm{Dy}$ & 799 & 104 & 105 & 109 & 113 \\
\hline${ }^{91} \mathrm{Zr}$ & 356 & 50 & 45 & 46 & 49 \\
\hline
\end{tabular}


The assumed limit value for $\mathrm{E}_{\mathrm{N}}$ plays an increasing role with the mass. There is a tendency for heavy nuclei to overestimate the data. The energy stored in nucleon emission, the collective modes such as vibrations, rotations and charge polarisations could partially fill this gap. Let us recall that the two-spheroid model correctly reproduces the total kinetic energy.

\section{Rotating hyperdeformed quasi-molecular states and critical angular momentum}

Rotating hyperdeformed states have been recently observed in Dy [50]. Ridges were found in $\mathrm{p}-\boldsymbol{\gamma}-\boldsymbol{\gamma}$ coincidence spectra corresponding to an extremely high dynamic moment of inertia of $130 \hbar^{2} . \mathrm{MeV}^{-1}$, suggesting the existence of strongly deformed prolate shapes of $\beta \geq 0.9$ in a high spin range of about $75-98 \hbar$. Very high dynamic moment of inertia of $140 \hbar^{2} . \mathrm{MeV}^{-1}$ has been also observed in Gd [51]. These experiments have been repeated and the data are still debated [52].

Calculations [53] based on the cranking Strutinsky method with a deformed Woods-Saxon potential had predicted the existence of hyperdeformed nuclear states but not for so high spins. Furthermore it had been rather assumed for Dy that hyperdeformed shapes would not likely be found because these states would not be populated below 70 $\hbar$, while above the fission decay mode would be dominant.

These rotating hyperdeformed states are populated in heavy-ion reactions (for example, $\left.{ }^{37} C+{ }^{120} S n \rightarrow{ }^{153} D y+1 p+3 n\right)$ and the starting configuration is two interacting spheres. Consequently, it is important to know the rotational dependence of the potential energy in the quasi-molecular shape valley which is particularly adapted to describe the entrance channel in heavy-ion collisions [54,55]. The 1-dependent barriers for Dy are displayed in Fig. 16. A scission barrier survives to spin $115 \hbar$ and macroscopic hyperdeformed states appear in a very spin range of about $70-110 \hbar$. The geometrical characteristics of these rotating isomeric states are for $1=70 \hbar, \beta \approx 0.8$, $I_{\perp} \approx 94 \hbar^{2} \cdot \mathrm{MeV}^{-1}$ and $Q \approx 20 \mathrm{eb}$. For a spin of $110 \hbar$, the values are $\beta \approx 0.96$, $I_{\perp} \approx 118 \hbar^{2} . \mathrm{MeV}^{-1}$ and $Q \approx 30 \mathrm{eb}$. The high values of the moment of inertia and curvature at the saddle-point explain that the centrifugal forces can remove the potential pocket only for very high angular momenta. The possibility to conciliate low fission barrier heights and high maximal angular momenta against fission is really a specificity of this deformation valley through quasi-molecular shapes. The first experimental data on hyperdeformed states in Dy are in agreement with these theoretical results but the knowledge of the lifetime and quadrupole moment is highly desirable and further experiments are needed.

The shell corrections are not included and one cannot obtain a potential well for $\beta$ $\approx 0.6$ explaining the superdeformed states observed experimentally. Nevertheless, these theoretical barriers seem also compatible with the available data on the superdeformed states in Dy : extension in spin from $22 \hbar$ to $60 \hbar$, top transition at an excitation energy of $30 \mathrm{MeV}$ and bands close to yrast at spins greater than $55 \hbar$. Furthermore, the stability against fission of these superdeformed states at $60 \hbar$ is ensured by a barrier of $14 \mathrm{MeV}$ in this deformation valley. 


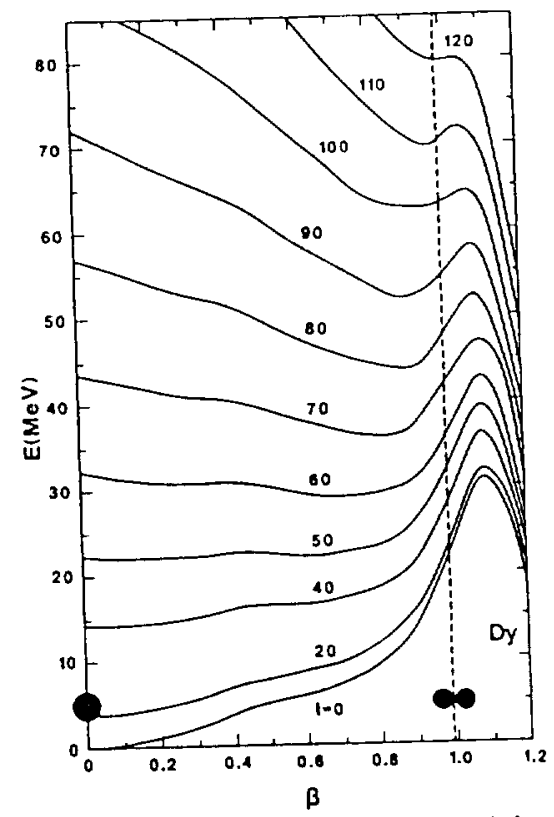

Fig. 16. Sum of the deformation and rotational energies as functions of the deformation and angular momentum ( $\hbar$ unit) for Dy. The dashed line indicates the separation into two equal spherical fragments.

In Fig. 17, the 1-dependent deformation energies are plotted for the ${ }^{24} \mathrm{Mg},{ }^{72} \mathrm{Se}$, ${ }^{132} \mathrm{Ce}$ and ${ }^{191} \mathrm{Hg}$ nuclei. The external barriers are high and thin and up to $\beta \approx 0.7-0.8$ the deformation energy profile is very flat at medium spins. The shape variation occurs at relatively constant energy and it is not surprising that, at intermediate angular momenta, shell rearrangements generate shallow wells where superdeformed states may survive. For lower spins and $\beta<0.7$ the slope of the potential energy curve is more pronounced and the shell effects will find it more difficult to dig a well. This might be the explanation for the observed sudden disappearance of the superdeformed states.
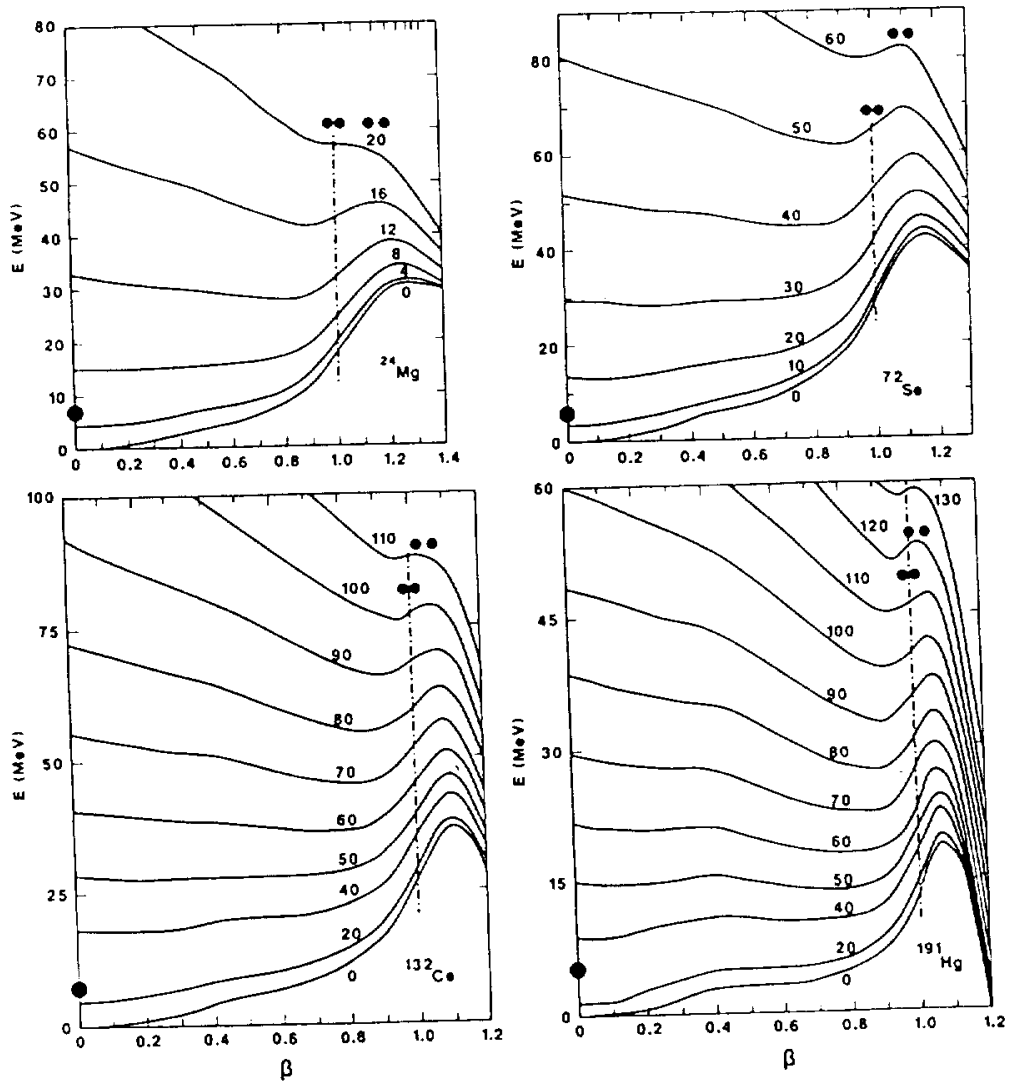

Fig. 17. Same as Fig. 16 but for ${ }^{24} \mathrm{Mg},{ }^{72} \mathrm{Se},{ }^{132} \mathrm{Ce}$ and ${ }^{191} \mathrm{Hg}$. 
With decreasing spins the external minimum located at the foot of the scission barrier moves to intermediate positions. Their geometrical characteristics are compared in Table 5 with data relative to the superdeformed states of $\mathrm{Ce}, \mathrm{Eu}, \mathrm{Dy}$ and $\mathrm{Hg}$. There is no evident contradiction and the macroscopic well at intermediate spins give a first rough indication on the superdeformed well emphasizing the importance of the underlying macroscopic potential surface.

Table 5. Experimental and theoretical quadrupole moment and moment of inertia for superdeformed states. The theoretical values correspond to the limit positions where the wells are still deeper than $0.5 \mathrm{MeV}$.

\begin{tabular}{|c|c|c|c|c|}
\hline nucleus & Exp Q (eb) & Theor $Q(e b)$ & Exp I $\left(\hbar^{2} \cdot \mathrm{MeV}^{-1}\right)$ & Theor I $\left(\hbar^{2} \cdot \mathrm{MeV}^{-1}\right)$ \\
\hline $\mathrm{Ce}$ & 8.8 & 10.4 & 55 & 60.5 \\
\hline $\mathrm{Eu}$ & 13 & 12.3 & 68 & 69.5 \\
\hline $\mathrm{Dy}$ & 19 & 14 & 85 & 79 \\
\hline $\mathrm{Hg}$ & 18 & 21.5 & 110 & 121 \\
\hline
\end{tabular}

To study the super and hyperdeformed states, the spheroidal configurations are often chosen even at very large deformations since the calculations of shell effects are easier. In Fig. 18, the characteristics of the deformation paths through compact and creviced shapes and prolate ellipsoidal shapes are compared. It is striking to observe that up to $\beta \approx 0.8-0.9$ the differences between the values of $I_{\perp}$ and $\mathrm{Q}$ in the two valleys are very small even though these two paths are so different [55]. Therefore, the values of $I_{\perp}$ and $\mathrm{Q}$ deduced from experimental data lead to a poor understanding of the nucleus elongation and neck development and the ellipsoidal and one-body nature of the strongly deformed rotating states is not proved.

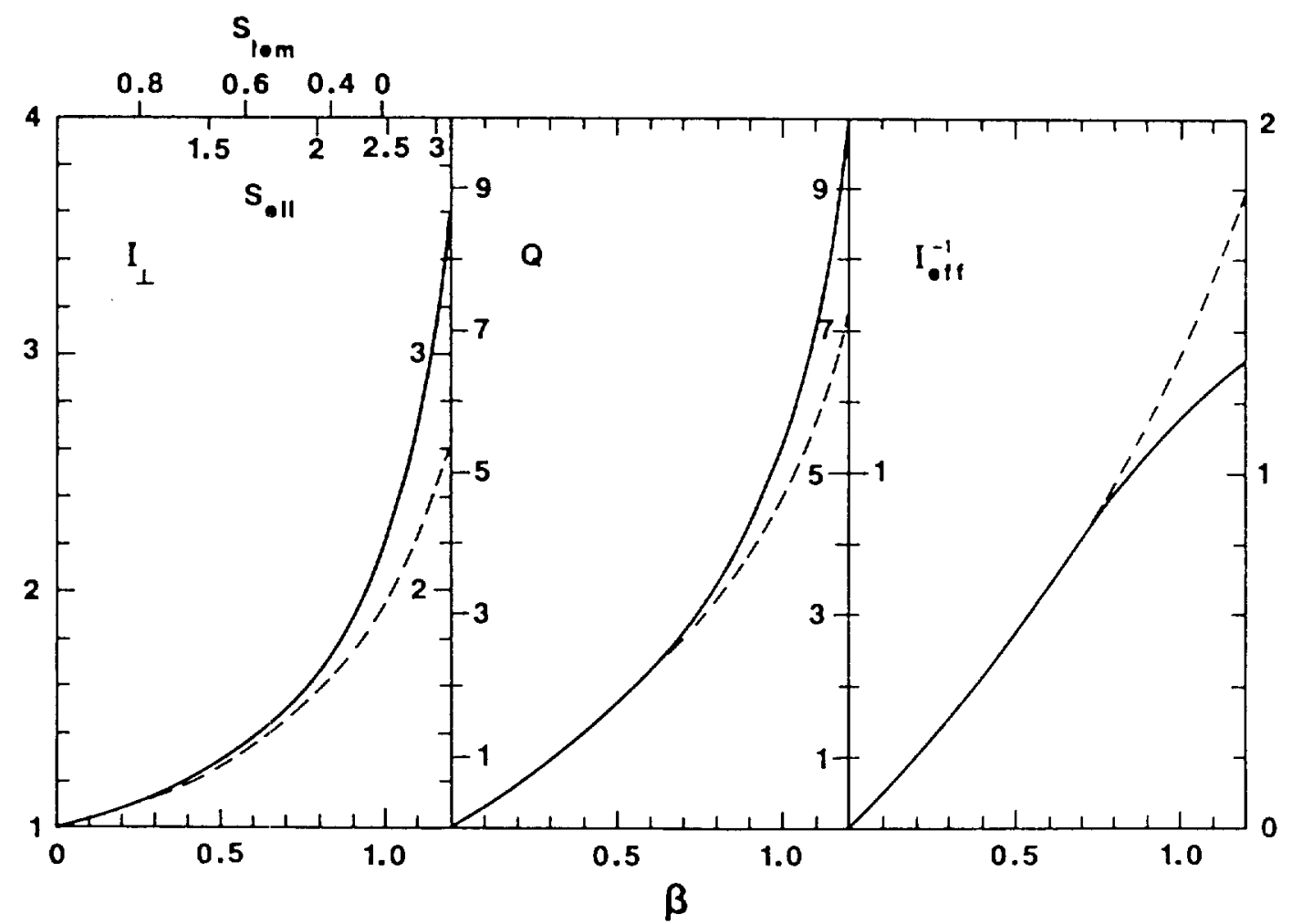

Fig. 18. Relative perpendicular moment of inertia, quadrupole moment and inverse effective moment of inertia of the elliptic lemniscatoids (full curve) simulating the quasi-molecular shapes and prolate spheroids (broken curve) as a function of the deformation. $S_{\text {lem }}$ is the ratio between the neck radius and half the elongation of the lemniscatoids while $S_{\mathrm{ell}}$ is the ratio between the axes of the ellipsoidal shapes. 
The critical angular momenta that light nuclei are able to sustain against the centrifugal forces have been obtained experimentally recently [56]. They are compared in Fig. 19 with calculations within the original version of the Liquid Drop Model [28], the Finite Range Liquid Drop Model [42] which uses a Yukawa plus exponential potential for the surface and proximity energies and the present generalized LDM applied to quasi-molecular shapes [57]. The pure liquid drop model overestimates the fission barrier heights and consequently the critical angular momentum. The $l_{c r}$ derived from the two approaches taking into account the nuclear proximity energy are in agreement with the experimental results.

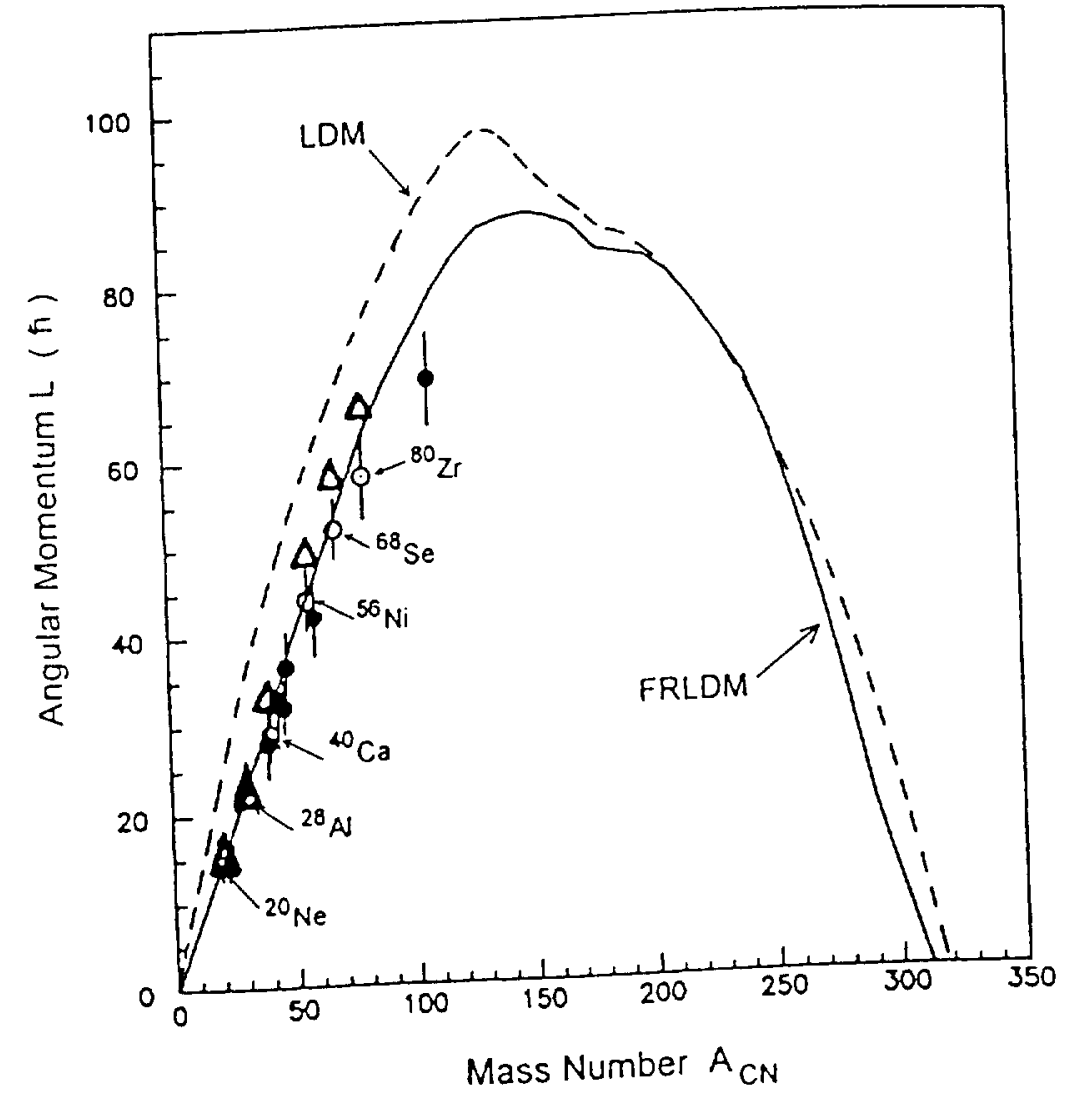

Fig. 19. Comparison of the experimental [56] critical angular momenta with theoretical predictions ( dashed curve [28], solid curve [42], triangles [57]) for light nuclei.

The question of the maximal angular momentum that medium and heavy nuclei are able to sustain against rotation is an old and still debated problem put forward once more by the new and questionable results at very high spins. In Fig. 20, three predictions of the critical angular momentum for $\beta$-stable nuclei are compared. Most of the studies [28, 42,58 ] predict a maximum of $l_{\text {cr }}$ of around $80-90 \hbar$, except Neergard and Pashkevich [59] who obtained around $110 \hbar$. Within our shape sequence and energy definition, the maximum of the critical angular momentum is approximately $130 \hbar$ for mass around 190. The question of the experimental value of $l_{\mathrm{cr}}$ is not simple since it can be reached only indirectly in fission processes following fusion reactions which are needed to bring enough angular momentum to the compound nucleus or via decay of hyperdeformed and superdeformed states. Nevertheless, the fast fission of semi-equilibrated fused systems at $l \approx 140 \hbar$ has been observed [60].

The agreement with the first data on hyperdeformed rotating states seems to confirm that the deformation valley through compact quasi-molecular shapes might be, really, a nuclear deformation valley. 


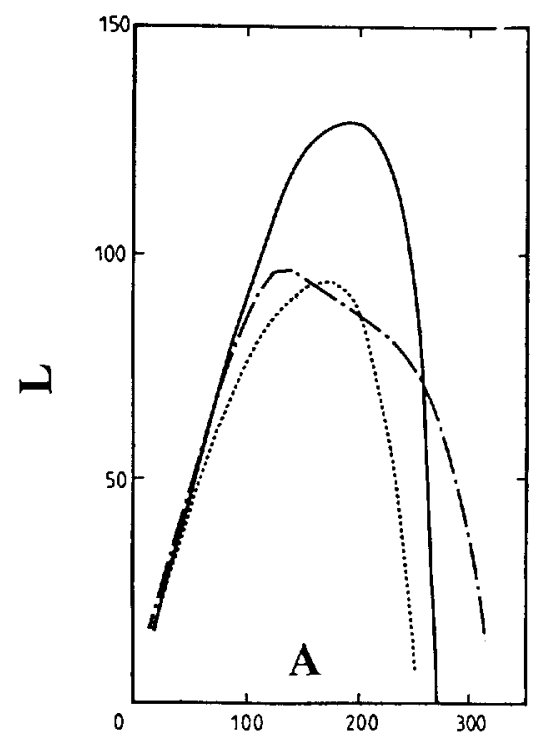

Fig. 20. Critical angular momentum ( $\hbar$ unit) for nuclei along the $\beta$ stability valley. The values obtained for quasi-molecular shapes are given by the full curve [22] while the theoretical predictions of Cohen et al [28] and Broglia et al [58] correspond respectively to the chain curve and the dotted curve.

\section{Ternary fission}

At low energies, the symmetric nuclear tripartition has an upper limit of $2 \times 10^{-9}$ relatively to binary fission, while for a lightest fragment in the mass range $A \geq 30$, the ratio is $8 \times 10^{-8}$. In contrast, very asymmetric ternary fission with the emission of an $\alpha$ particle occurs with a relative yield of $3-6 \times 10^{-3}[61,62]$. Ternary fission was also expected in the reaction ${ }^{238} U+{ }^{238} U \rightarrow{ }_{184}^{476} S h$ but has not been observed.

At intermediate energies (10-100 MeV/A), highly excited systems are obtained by partial fusion of the projectile and the target and a large amount of thermal excitation energy and rotational energy is deposited. Besides particle emission and binary fission, the nuclear ternary fragmentation is also an important decay mode for these hot systems [63].

The ternary fission valley through elongated shapes with wide necks where the proximity forces are negligible has been extensively investigated [64]. On the other hand, the family of quasi-molecular shapes previously used in the binary case has been generalised to describe and study [65-67] the prolate ternary fission (see Fig. 2). It covers the whole deformation range from the sphere to three aligned tangential spherical nuclei; the two external fragments being identical.

The different contributions to the potential energy are given separately together with the deformation energy in Fig. 21 for the symmetric and extreme asymmetric ternary fission for ${ }^{212} \mathrm{Po}$. In Fig. 22, the barriers are given for a light nucleus and an heavy one for different asymmetries [68]. As in the binary case, the total energy varies gently even around the contact point where the slope of the $E_{s}$ and $E_{n}$ curves changes drastically and the nuclear attraction is the greatest. For light nuclei and for all the mass asymmetry values, the saddle point corresponds to three separated fragments held together by the proximity forces which counterbalance the Coulomb repulsion and the potential barrier is a scission barrier. For heavier nuclei, the saddle point shape has the same configuration, except for very high asymmetries ( $\alpha$ particle emission for example) 
where the barrier top corresponds to a one-body compact shape encountered before the formation of the necks since the proximity energy is not sufficient to introduce an inflection in the potential curve. The height of the barrier depends strongly on the decay asymmetry.

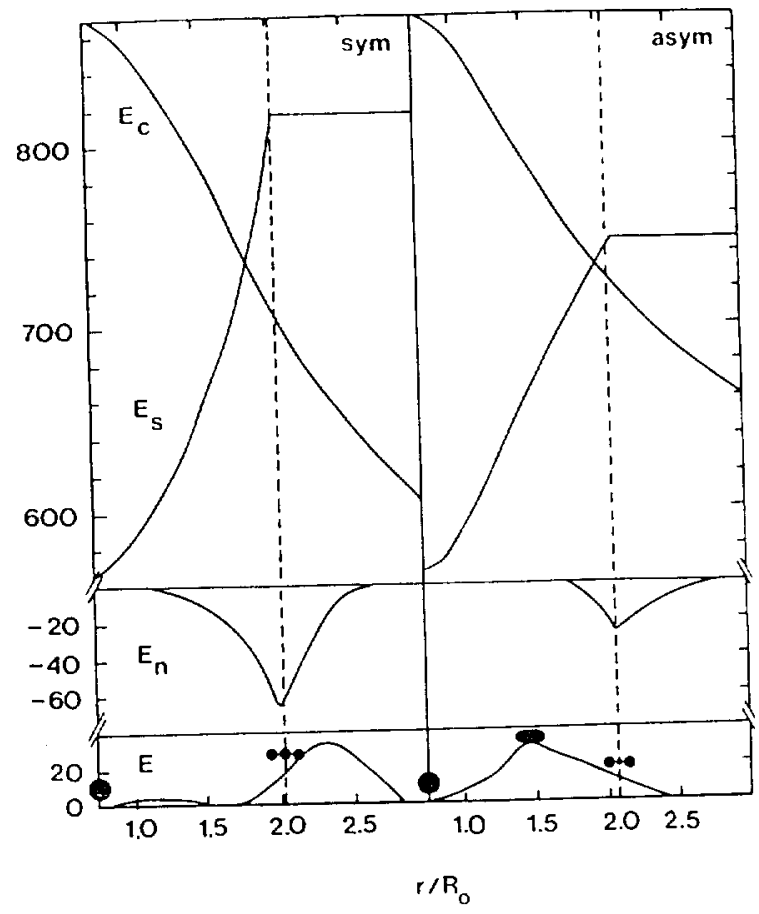

Fig. 21. Variation of the surface energy $E_{s}$, the Coulomb energy $E_{c}$, the nuclear proximity energy $E_{N}$ and the deformation energy $\mathrm{E}$ (in $\mathrm{MeV}$ ) for the ${ }^{212} \mathrm{Po}$ nucleus plotted against the reduced distance between the mass centres (of the two halves of the system [65]) in the path of the ternary fission through compact and creviced shapes. The broken line corresponds to the separation into three spherical fragments. The central nucleus is an alpha particle for the asymmetric configuration (right column)
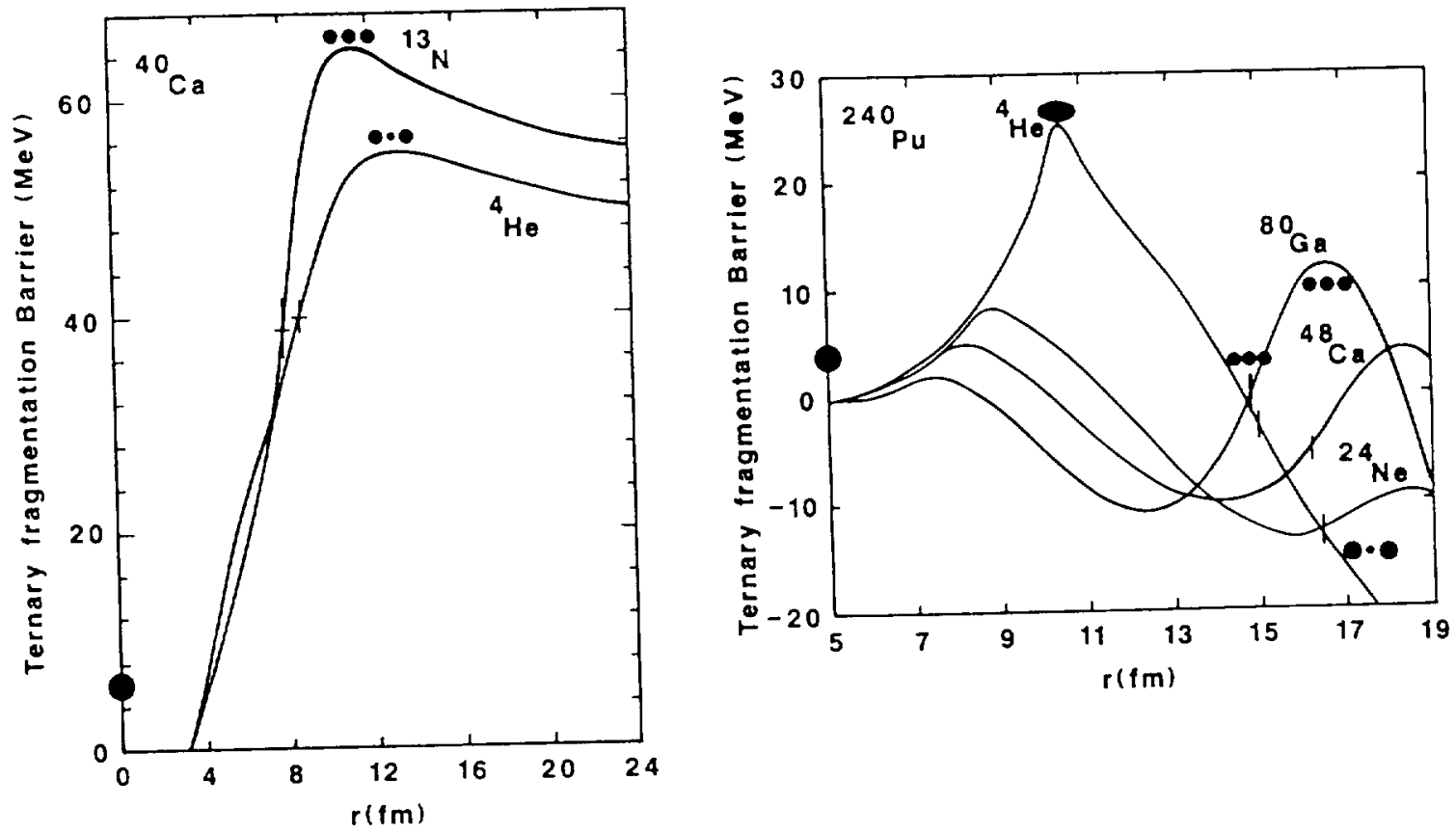

Fig. 22. Ternary fission barriers as a function of the distance $r$ between the mass centres for the ${ }^{40} \mathrm{Ca}$ and ${ }^{240} \mathrm{Pu}$ nuclei. The central fragment is indicated on the curves and the separation point by a vertical bar.

In Fig. 23, binary and ternary symmetric fission barriers are compared. The two barriers have the same structure : one peak for medium nuclei and two peaks separated 
by a deep minimum for heavier nuclei. A greater elongation in the ternary case causes the outer peak of the ternary fission barrier to be always further out than in the binary case. In this mass range, the ternary fission barrier is much higher than the binary one and ternary fission is very energetically unfavourable, although the formation of threebody isomeric states corresponding to the deep minimum of the ternary fission barrier seems possible. For $Z>102$, the ternary fission barrier is lower than the binary one but it remains further out. The height of the second peak relatively to the second minimum is important for $Z<110$ but this second barrier lies well below the first peak. The hypothesis that the fragment kinetic energy corresponds to the interaction Coulomb energy at the external scission point where $\left|E_{N}\right|<0.2 \mathrm{MeV}$ allows to roughly reproduce the kinetic energy of the three comparable fragments detected in the U+Ne and $\mathrm{U}+\mathrm{Ar}$ reactions $[65,69]$.
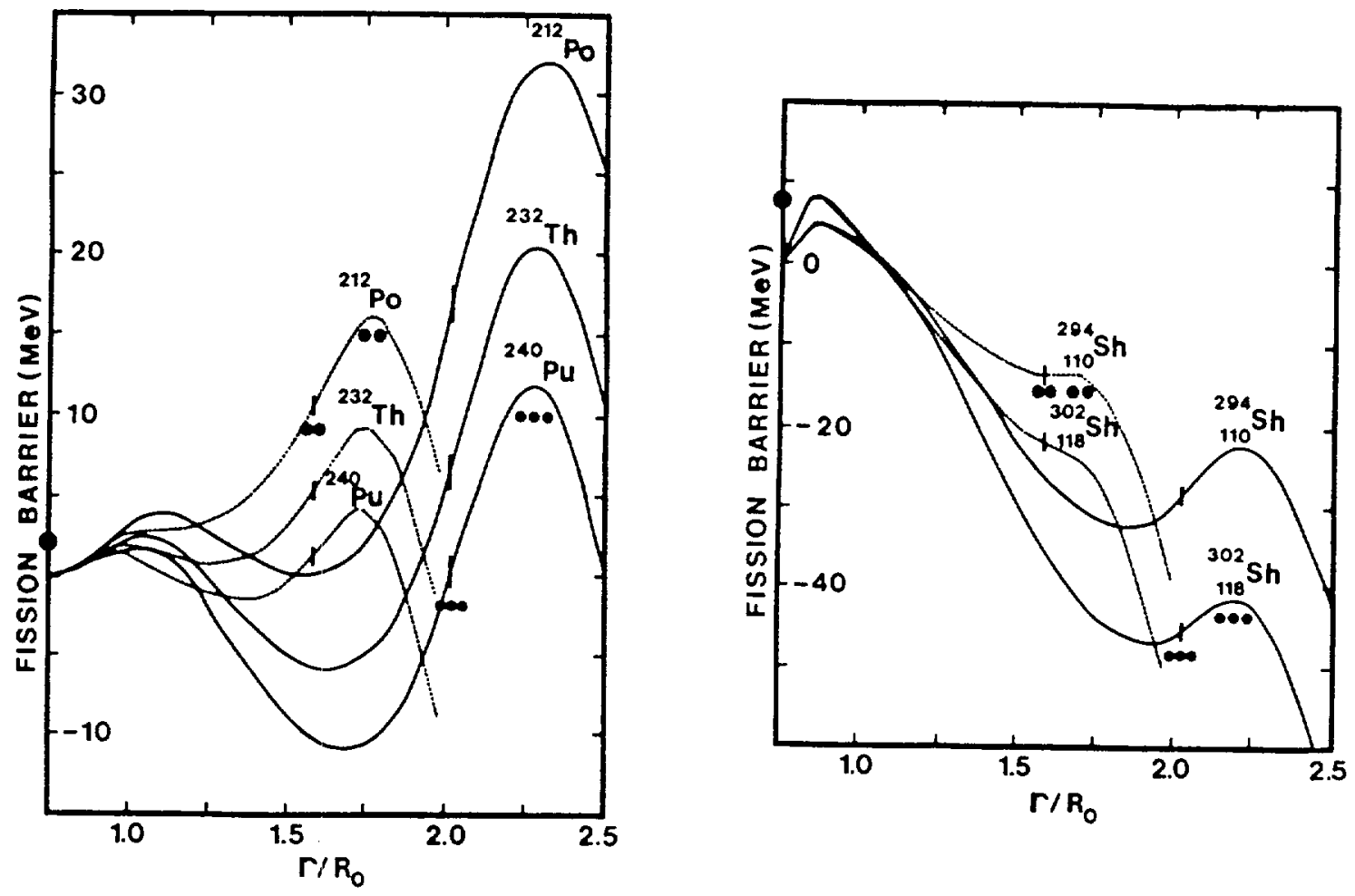

Fig. 23. Binary (dotted curves) and ternary (full curves) symmetric fission barriers for the nuclei ${ }^{212} \mathrm{Po},{ }^{232} \mathrm{Th}$, ${ }^{240} \mathrm{Pu},{ }_{110}^{294} \mathrm{Sh}$ and ${ }_{118}^{302} \mathrm{Sh}$ plotted against the reduced distance between the two halves of the fissioning system. The shell effects have been introduced for the superheavy elements within the Droplet model prescription. The vertical bars indicate the contact point.

The macroscopic fission barrier heights for binary and ternary fission are compared in Fig. 24. The ternary fission may occur only for very heavy and very light nuclei. For heavy systems the two tails of the curve show that the whole structure of the doublehumped ternary fission barrier must be examined and not just the barrier height relatively to the ground state.

Recently, the fission of ${ }^{48} \mathrm{Cr}$ formed in fusion reactions has been reported [70] and the l-dependent binary and ternary fission barriers are compared in Fig. 25. It is quite striking to observe that the deformation energy of the minimum in the 25-40 $\hbar$ range is of the same order or lower in the symmetric ternary decay channel than in the binary one and that its position is more external (higher moment of inertia). Then, one may not exclude the occurrence of prolate ${ }^{16} \mathrm{O}+{ }^{16} \mathrm{O}+{ }^{16} \mathrm{O}$ molecular configurations following fusion reactions in this spin and excitation energy range [71]. Analyses of experimental results to test this hypothesis are in progress [72]. 


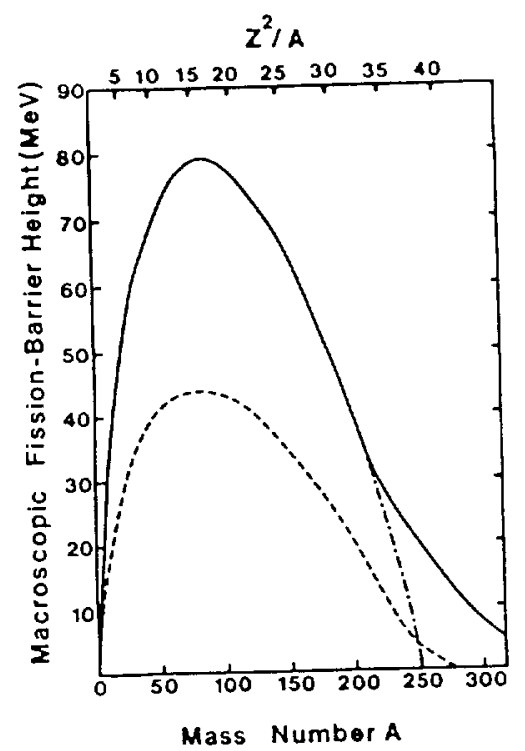

Fig. 24. Macroscopic fission barrier height for binary fission (broken curve) and ternary fission in the $\beta$ stability valley. For ternary fission, the chain curve gives the height of the outer maximum of the double-humped barrier relatively to the ground state while the full curve displays the height of the external peak relatively to the outer minimum of the barrier.
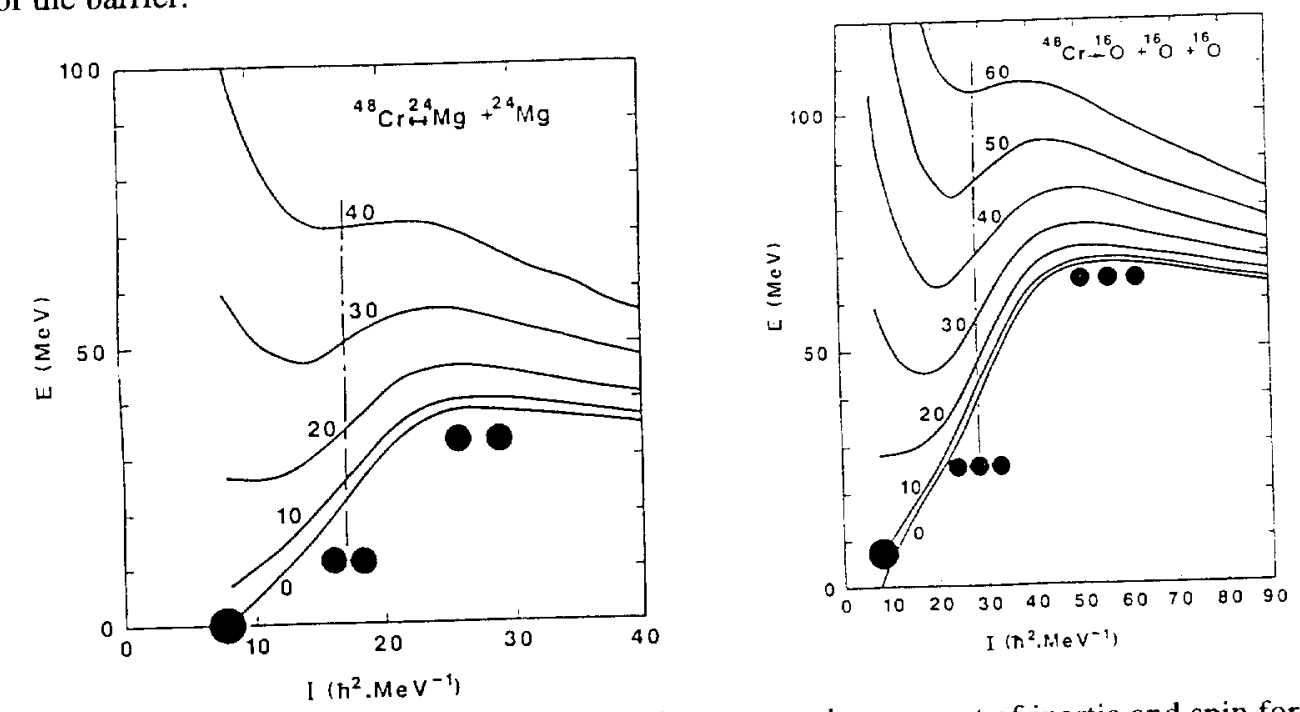

Fig. 25. Binary and ternary symmetric fission barriers versus the moment of inertia and spin for ${ }^{48} \mathrm{Cr}$

\section{Influence of the temperature and rotation}

The new generation of accelerators built in laboratories such as GANIL, GSI, MSU, TexasAM, HMI and SATURNE provides heavy-ions in the intermediate energy domain (10-100 MeV/A) allowing the study of highly excited nuclei. These systems are obtained by partial fusion of the projectile and the target and a large amount of thermal excitation energy and rotational energy is deposited. Pairing effects vanish around $\mathrm{T}=1$ $\mathrm{MeV}$ while shell effects disappear at $\mathrm{T}=2-3 \mathrm{MeV}$. Particle emission is enhanced with excitation energy. It has been clearly established that fusion still exists at $60 \mathrm{MeV} / \mathrm{A}$ and that nuclear systems can be formed in thermal equilibrium up to temperature as high as 5

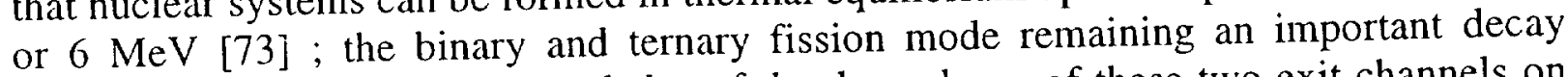
channel. Consequently, the knowledge of the dependence of these two exit channels on the temperature, angular momentum, mass and asymmetry is important $[41,68]$.

The dependence on the temperature of the binary and ternary fission barrier heights are displayed in Fig. 26. The heating of a nucleus strongly lowers its fission barrier and 
the lowering is all the more important as the system is heavy since the main temperature effect is to diminish the surface tension. At $\mathrm{T}=5 \mathrm{MMeV}$, the binary fission barriers vanish for $A-200$. In the ternary fission valley, due to the high external peak persisting for high masses, quasi-molecular isomeric states are able to sustain high temperature.
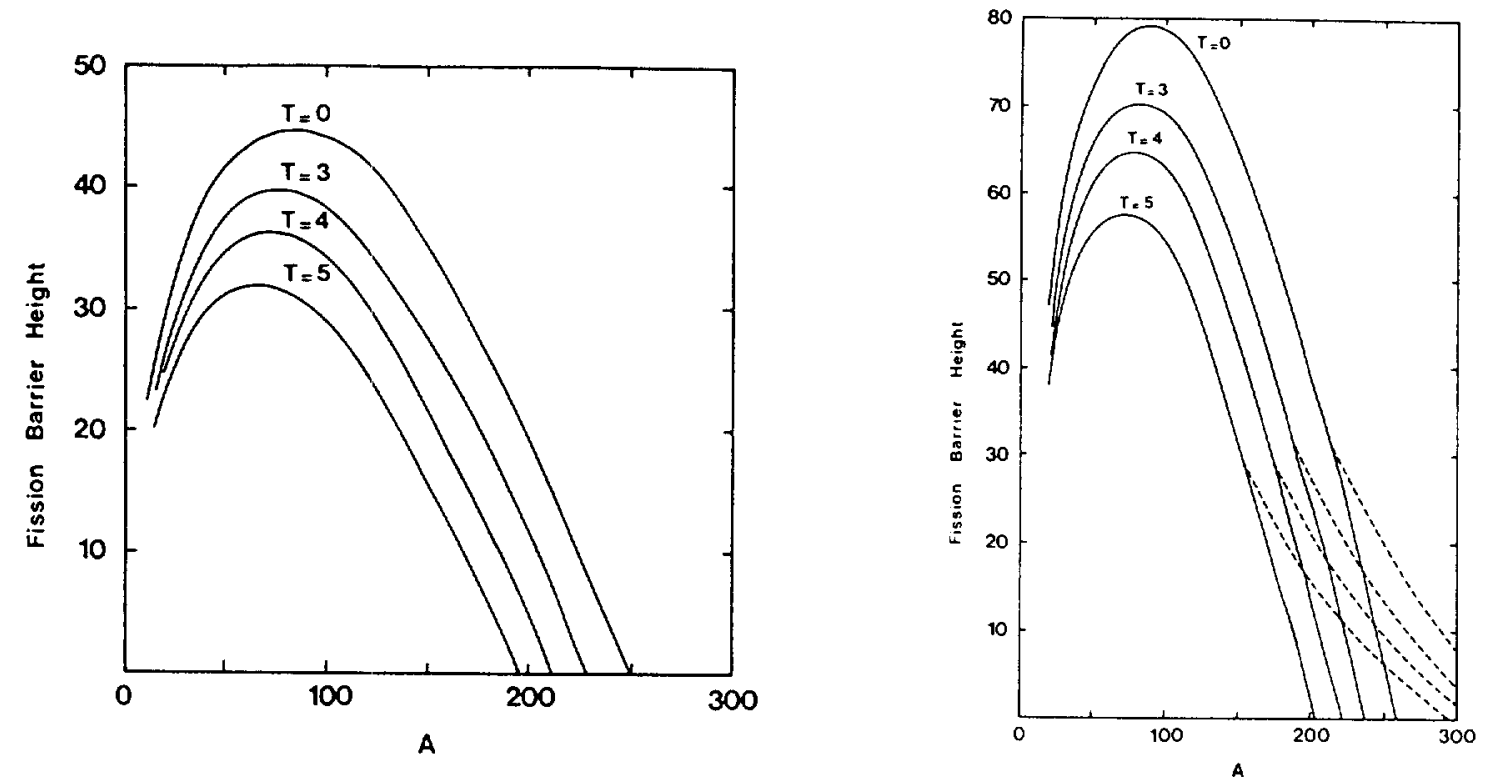

Fig. 26. Barrier heights (MeV unit) for the symmetric binary (left part) and ternary (right part) fission of nuclei along the $\beta$ stability valley as a function of the temperature $(\mathrm{MeV}$ unit) and the mass number $(l=0)$. The broken curves correspond to the height of the external peak relatively to the second minimum of the barrier.

The decrease of the binary fission barrier heights when a nuclear system is heated and rotates is shown for four selected typical nuclei in Fig. 27. The differences between mass inertia explain that the barrier height for light nuclei vanishes rapidly for low angular momenta, while for heavy systems, centrifugal forces compensate for the deformation energy only for very high angular momenta. For all systems, the temperature dependence of the barrier height diminishes when the angular momentum increases.
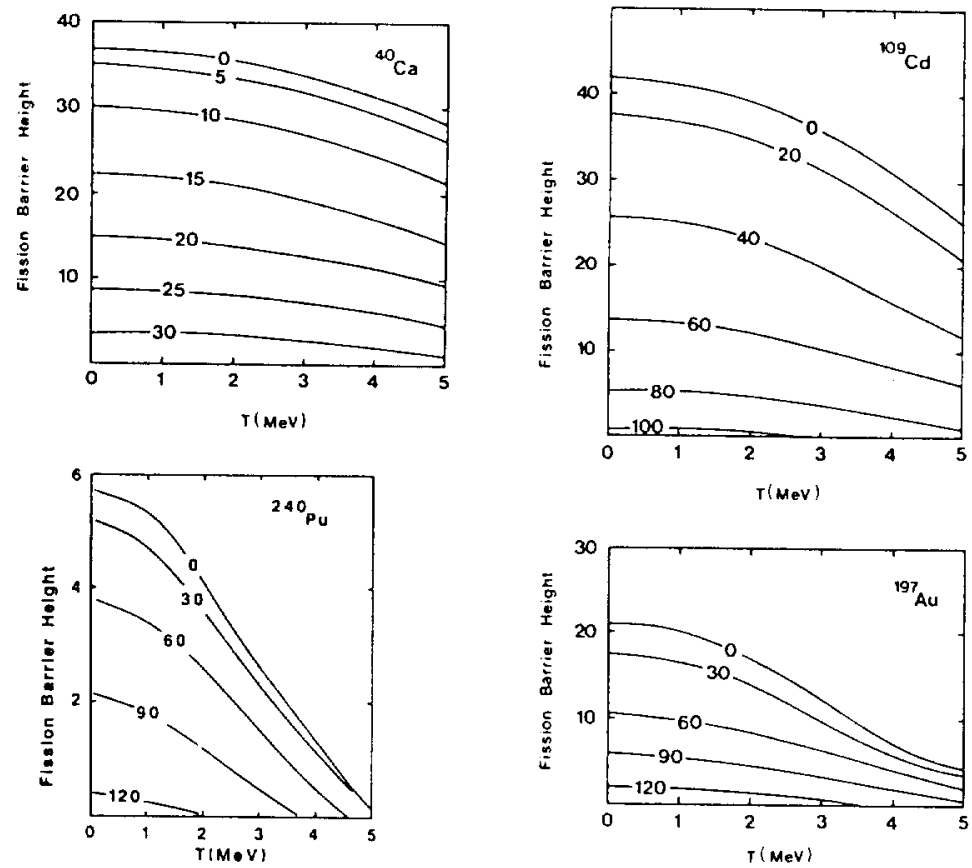

Fig. 27. Binary and symmetric fission barrier heights (MeV unit) as functions of the temperature and the angular momentum ( $\hbar$ unit) for ${ }^{40} \mathrm{Ca},{ }^{109} \mathrm{Cd},{ }^{197} \mathrm{Au}$ and ${ }^{240}$ nuclei. 
The ridge-line potentials for the four above mentioned nuclei located below and above the Bus.naro-Gallone point are displayed in Fig. 28. The lowering of the surface energy and, then, of the fission barrier height by the temperature increases with the symmetry of the system and with its mass. The incorporation of temperature maintains essentially the same topology of the Businaro-Gallone picture : below the BG point the ridge line shows a maximum at symmetry while above the symmetric saddle-point is stable with respect to the mass-asymmetry mode and is the ordinary fission saddle-point. Nevertheless, there is a shift of the BG point toward lower fissility values as the temperature increases and also as the angular momentum increases. High temperature and rotation favour symmetric fission.
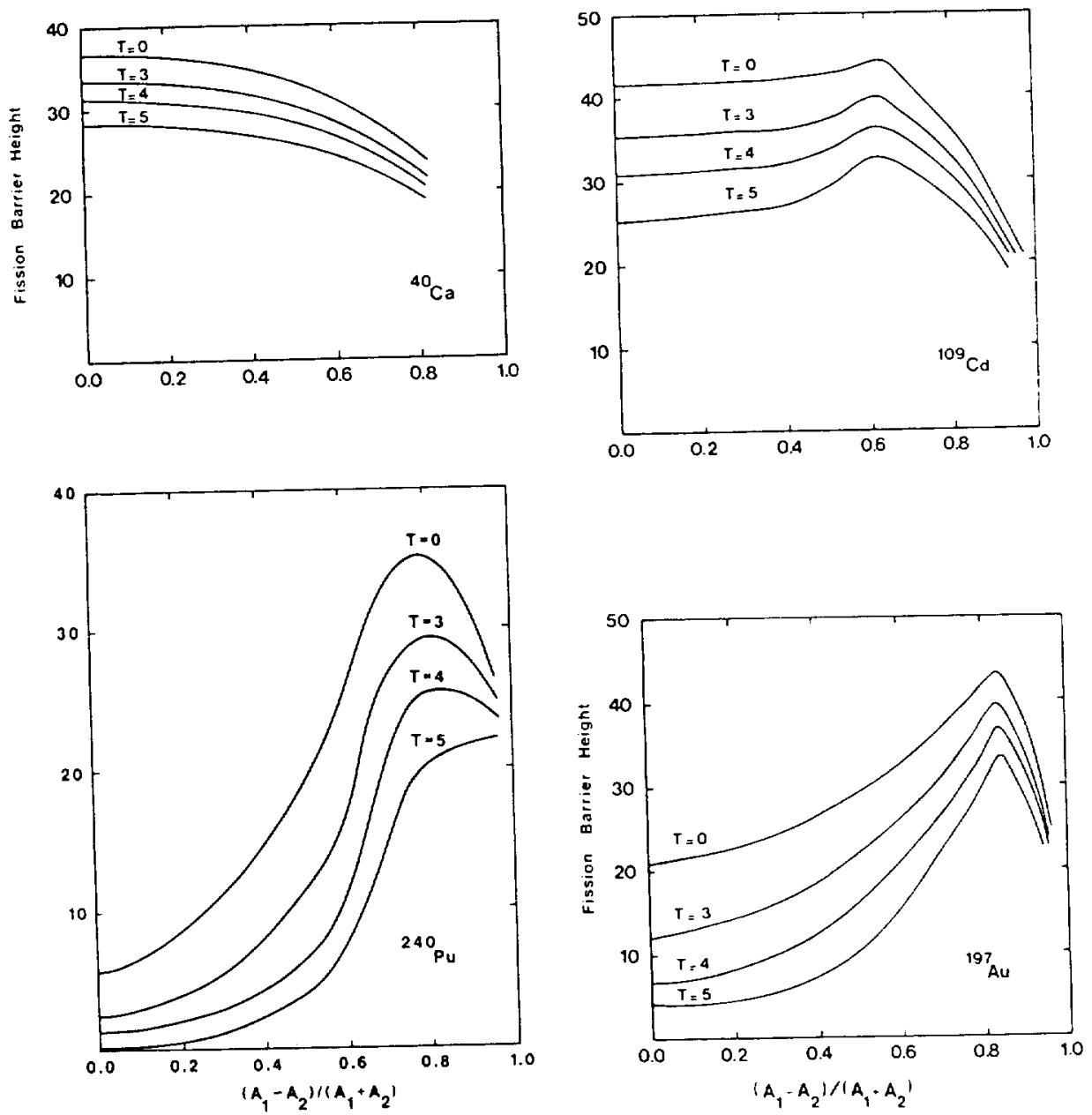

Fig. 28. Binary fission barrier heights (MeV unit) as functions of the temperature and the decay asymmetry for the ${ }^{40} \mathrm{Ca},{ }^{109} \mathrm{Cd},{ }^{197} \mathrm{Au}$ and ${ }^{240}$ nuclei. $\mathrm{A}_{1}$ and $\mathrm{A}_{2}$ are the masses of the two nascent fragments.

\section{Fragmentation and formation of bubbles and toroids}

In heavy-ion collisions at 30-100 MeV/nucleon, although binary and ternary fission remains an important exit channel, quaternary, quinary,... decays have been observed at high excitation energies [74]. The interpretation of this multifragment production is still elusive. Several explanations have been advanced $[75,76]$ including : dynamically induced density fluctuations, expansion of an initially compressed source, statistical decays and rapid sequential binary fission. Starting from the idea that the fragments are emitted by an almost thermalized system, the fragmentation barriers into several spherical fragments emitted in a volume-like manner [77] and in a ring-like 
manner [78] have been calculated within symmetric quasi-molecules. The selected geometric configurations are respectively for the fragment mass centres : equilateral triangle, tetrahedron, square, spheres along three cartesian axes, hexagon, cube and octagon. The starting configuration is $\mathbf{n}$ touching spheres.

These barriers of fragmentation in $\mathrm{n}$ nuclei are compared in Fig. 29 for ${ }^{240} \mathrm{Pu}$. Obviously, the binary and oblate ternary decay paths are the same in the two geometrically different fragmentations. In all cases, the barrier tops correspond to $\mathrm{n}$ separated fragments maintained in unstable equilibrium by the balance between the repulsive Coulomb forces and the attractive nuclear forces and so generalising the picture observed for the binary decay through quasi-molecular shapes. The fragmentation barriers are lower when the emission is focalized in a same plane since the proximity forces act at larger deformations and, then, for smaller Coulomb repulsion. Then, even if crude hypotheses have been asserted, our calculations indicate that the emission of the fragments in the same plane is somewhat favoured. In the case of the three dimensional emission, the barrier heights increase strongly with the number of fragments even for very high temperature. For the plane multifragment emission, the situation is reversed [78] for the heaviest systems (obtained as evanescent residues in reactions such as $\mathrm{Gd}+\mathrm{U}, \mathrm{Pb}+\mathrm{Au}, \mathrm{Au}+\mathrm{Au}$ ). Indeed, apart from the binary fission mode, the potential barrier heights become comparable and even decrease slightly with the number of fragments.

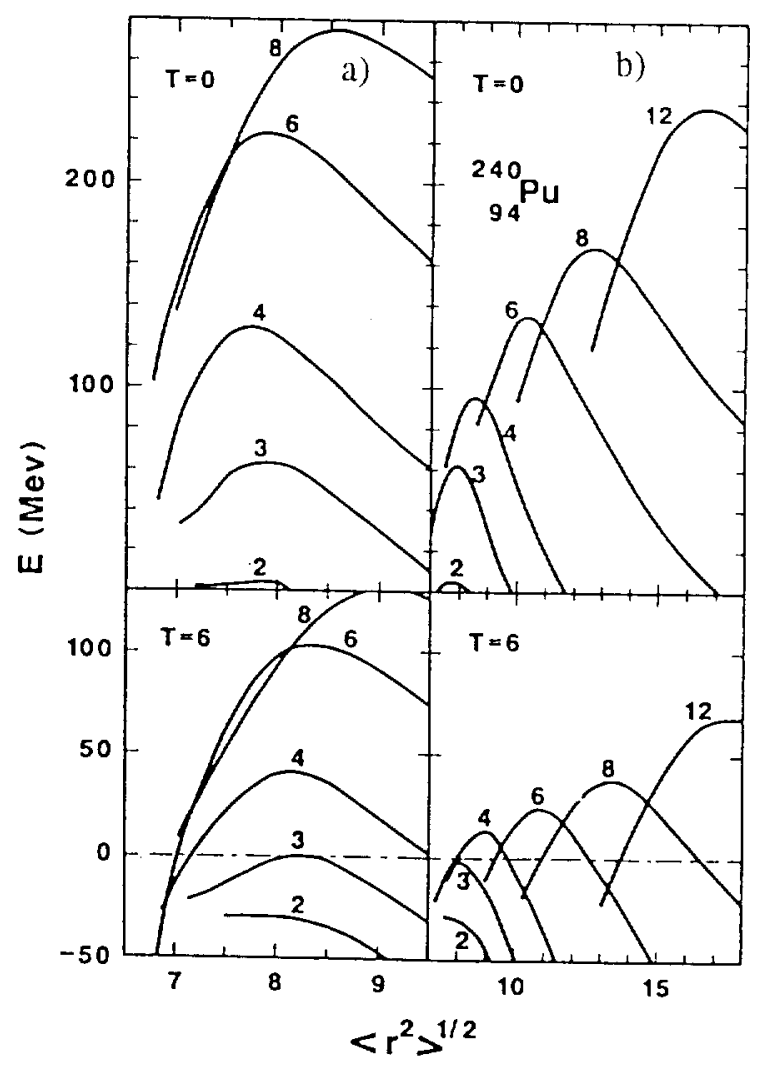

Fig. 29. Fragmentation barriers (potential energy relatively to the initial sphere energy) as functions of the number of fragments and the temperature (MeV unit) for the ${ }^{240} \mathrm{Pu}$ nucleus. The left column a) corresponds to the cmission of fragments in the whole space while the right column b) is relative to the fragment emission in a plane.

From recent experiments for intermediate mass systems, it has been suggested [9] that a statistical competition between the various multifragmentation channels occurs. For all the excitation energies, the $n$ event probabilities decrease with the fragment 
number but the yield of threefold, fourfold and fivefold events increases with the excitation energies. This behaviour can be understood looking at the fragmentation barriers. At low excitation energies, the barrier height plays the major role and binary and ternary fission is dominant. In contrast, for high excitation energies, only a small part of the available energy is absorbed during the heating of the residual system while the other remaining and non-thermalized part is so important that the investigation of all the fragmentation channels is possible. The situation is different for the heaviest systems. All the multifragmentation events in the same plane seem to have roughly the same probability even for low excitation energies. Nevertheless, the problem to form these very evanescent heaviest mixtures of matter with no binary fission barrier is not simple since it is necessary to overcome the deep-inelastic regime and low excitation energies are perhaps not sufficient.

Some recent experimental data [79] and simulations within the BoltzmannUehling-Uhlenbeck model [80] seem to point out that effectively, for very violent collisions of intermediate mass systems, the thermal source expands rather in a plane perpendicular to the line connecting the centres of projectile and target. This transverse focalization is mainly due to the initial compression which induces strong oblate deformations in which Rayleigh instabilities develop allowing the plane multifragment emission.

When the compression effects and the Coulomb repulsion are extremely important as in violent central collisions of very massive nuclei, calculations have shown that a depression of matter might occur in the centre of the distribution of nucleons just after the most violent phase of the reaction [81-83]. Recently, first experimental signatures in very massive collisions like $\mathrm{Pb}+\mathrm{Au}[84,85]$ seem to indicate that evanescent and strongly deformed shapes intermediate between toroidal and bubble configurations develop before the fragmentation in several intermediate mass fragments. These exotic topologies were early on suggested as being metastable long time ago.

The energy of a nuclear system evolving in the deformation path leading continuously from an initial spherical shape towards toroidal configurations and bubbles has been studied within the above described generalized liquid drop model and selected shape sequences $[86,87]$. The toroidal deformation barriers and barriers of plane fragmentation are displayed for ${ }^{322} 128$ in Fig. 30 while, in Fig. 31, the comparison also includes the bubble deformation barriers and barriers of three dimensional emission for ${ }^{400} 147$. For these very heavy systems, a wide and deep potential pocket appears in the toroidal deformation path. Furthermore, this well is located below the potential barriers for the $\mathrm{n}$ fragment emission in some deformation range. This might perhaps allow to such exotic toroidal shapes, generated by the dynamics in the first phase of the most massive heavy-ion collisions, to survive in a metastable state before decaying in the multifragment exit channels due to the effects of the surface tension forces which clusterize the matter distribution to minimize the nuclear surface. The angular momentum transfer accompanying the formation of these toroidal shapes in heavy-ion reactions might also slightly increase their stability. In contrast, the three dimensional fragmentation barriers are well below the bubble-like deformation barriers whatever the mass and the temperature of the system are. So, if the dynamics in the most massive central heavy-ion collisions leads the nuclear system to very distorted rotating hollow bubble configurations after the compression phase, the system will afterwards evolve rather to evanescent toroidal matter distributions while clusterising under the action of the surface tension and proximity forces. 


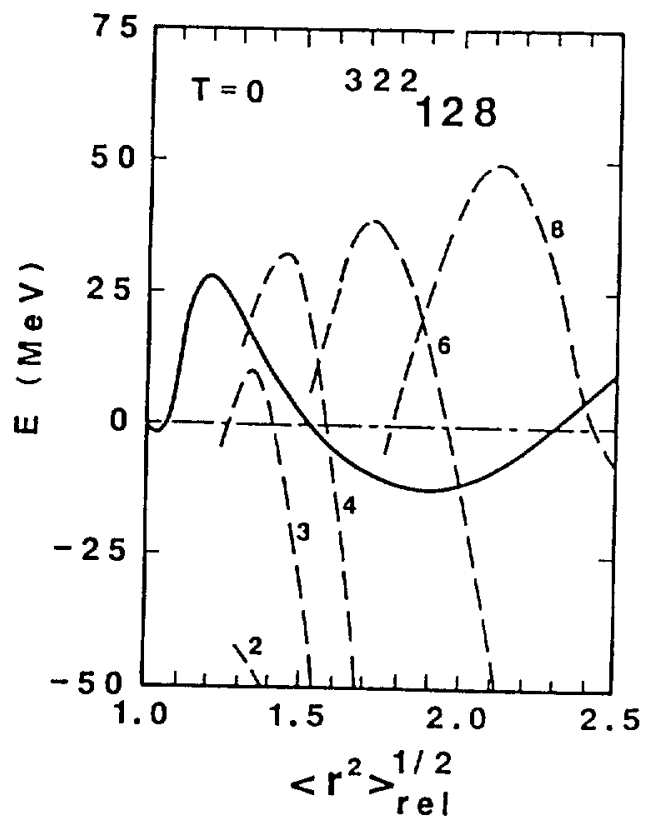

Fig. 30. Comparison between the toroidal deformation barrier (full curve) and barriers of plane fragmentation into $2,3,4,6$ and 8 fragments (dashed curves) for ${ }^{322} 128$.

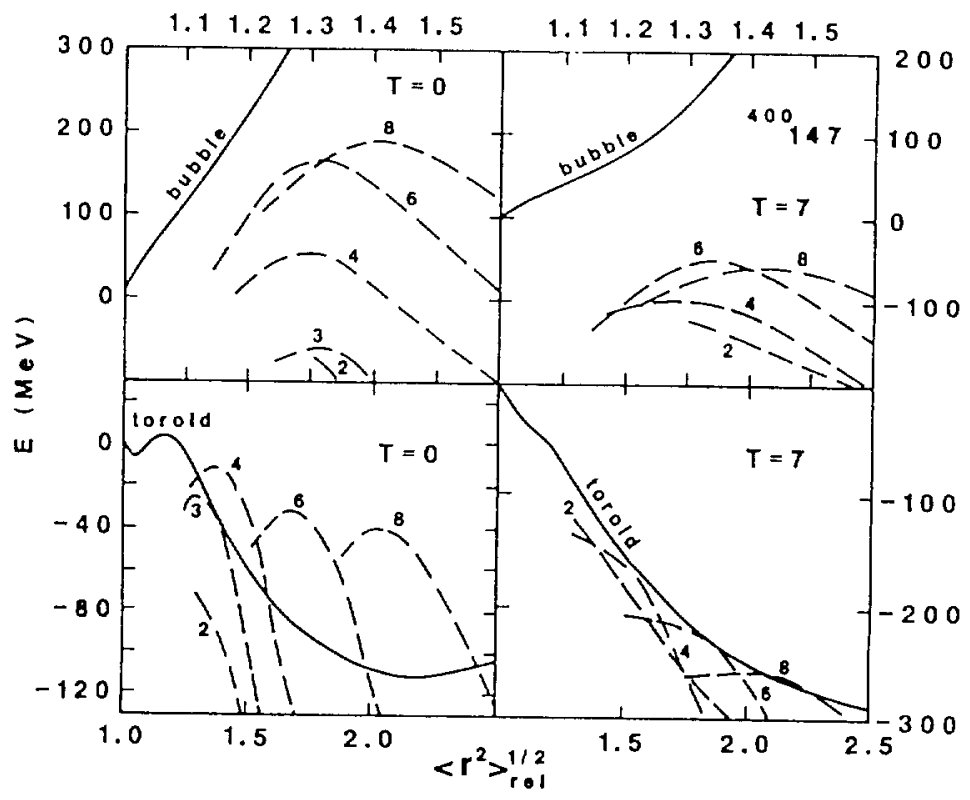

Fig. 31. Comparison at $\mathrm{T}=0$ and $7 \mathrm{MeV}$ between the bubble deformation barriers (full curves) and barriers of three dimensional emission (dashed curves) in the upper part of the figure and between the toroidal deformation barriers (full curves) and barriers of plane fragmentation (dashed curves) in the lower part for ${ }^{400} 147$.

\section{Conclusion}

The decay path which leads an initial spherical nucleus through compact and creviced shapes with almost spherical ends and to two or more spherical tangent fragments which later go away has been investigated within a generalized liquid drop model taking into account both the proximity energy, an accurate sharp radius, the decay asymmetry and the temperature effects. The original value of the surface coefficient has been maintained. 
Within this approach of the nuclear deformation energy, this exit channel through quasi-molecular shapes is compatible with most of the experimental data : symmetric fission barrier heights in the whole mass range, asymmetric fission barrier heights of In, $\mathrm{Tb}$ and Mo, Businaro-Gallone point, double-humped barriers of actinides, fragment kinetic energies, partial half-lives of radioactive nuclei emitting heavy clusters and critical momenta for light and medium nuclei. The rotational hyperdeformed states recently observed might also come up and survive in this fusion-like fission valley. The rotation as well as the thermal excitation favour the symmetric splitting. In this peculiar decay path, the fission barrier is a scission barrier hindering the rupture of the neck between the nascent fragments. The nature of the saddle-point is unusual, it corresponds to separated fragments maintained in metastable equilibrium by the balance between the attractive proximity forces and the repulsive Coulomb forces. Analytical formulas given the fission barrier characteristics in this deformation path will be provided in a close future [88].

The fragmentation barriers for emission in a plane or in the whole space as well the deformation energies of toroids and bubbles have been determined. For very heavy systems, minima lying below the barriers of plane fragmentation exist in the toroidal deformation valley allowing some stability and relaxation of the excited toroidal system before its decay in several fragments emitted in a plane due to the surface tension and proximity forces.

\section{Acknowledgments}

I thank warmly my colleagues : V. Yu. Denisov, E. Druet, C. Fauchard, Raj K. Gupta, F. Haddad, B. Jouault, J. Mignen, C. Normand, Y. Raffray and B. Remaud.

\section{References}

[1] F. Gönnenwein, in The Nuclear Fission Process, ed. C. Wagemans (CRC Press Inc., Boca-Raton, Florida, 1991) p. 287.

[2] F.J. Hambsch and S. Oberstedt, Nucl. Phys. A 617 (1997) 347.

[3] R K. Gupta and W. Greiner, Int. J. Mod. Phys. E 3 (1994, Supp.) 335.

[4] A. Guglielmetti et al, Phys. Rev. C 52 (1995) 740.

[5] M. Freer, N.M. Clarke, R.A. Le Marechal, G. Tungate, R.P. Ward and W.D.M. Rae, Phys. Rev. C 51 (1995) 3174

[6] J. Boger and J.M. Alexander, Phys. Rev. C 50 (1994) 1006.

[7] N.V. Antonenko, E.A. Cherepanov, A.K. Nasirov, V.P. Permjakov and V.V. Volkov, Phys. Rev. C 51 (1995) 2635

[8] J.F. Lecolley et al, Phys. Lett. B 325 (1994) 317

[9] W.G. Lynch, Nucl. Phys. A 583 (1995) 471c.

[10] S. Cohen and W.J. Swiatecki, Ann. of Phys. 22 (1963) 406

[11] V.M. Strutinsky, N.Ya. Lyashchenko and N.A. Popov, Nucl. Phys. 46 (1963) 639.

[12] J.R. Nix, Ann. Rev. Nucl. Sci 22 (1972) 65.

[13] M. Brack, J. Damgaard, A.S. Jensen, H.C. Pauli, V.M. Strutinsky and C.Y. Wong, Rev. Mod. Phys 44 (1972) 320.

[14] J.F. Berger, M. Girod and D. Gogny, J. Physique Lett 42 (1981) 509

[15] A.H. Blin and B. Hiller, Nucl. Phys. A 391 (1982) 505

[16] J. Blocki, J. Randrup, W.J. Swiatecki and C.F. Tsang, Ann. of Phys. 105 (1977) 427.

[17] H. Feldmeier, $12^{\text {th }}$ Summer School on Nuclear Physics, Mikolajki, Poland, 1979.

[18] B. Leroux, G. Barreau, T. Benfoughal, B. Bruneau, F. Caitucoli, N. Carjan, T.P. Doan and A. Sicre, J. Physique Coll 41 C3 (1980) 133.

[19] G. Royer and B. Remaud, Nucl. Phys. A 444 (1985) 477. 
[20] G. Royer, J. Phys. G 12 (1986) 623.

[21] W.D. Myers and W.J. Swiatecki, Ark. Phys. 36 (1967) 343.

[22] G. Royer and B. Remaud, J. Phys. G 10 (1984) 1057.

[23] R.W. Hasse and W.D. Myers, Geometrical Relationships of Macroscopic Nuclear Physics (Springer, Berlin, 1988).

[24] H.J. Krappe, J.R. Nix and A.J. Sierk, Phys. Rev. C 20 (1979) 992.

[25] W.D. Myers and W.J. Swiatecki, Nucl. Phys. A 601 (1996) 141.

[26] G. Royer and B. Remaud, J. Phys. G 8 (1982) L159.

[27] J.R. Nix and W.J. Swiatecki, Nucl. Phys. 71 (1965) 1.

[28] S. Cohen, F. Plasil and W.J. Swiatecki, Ann. of Phys. 82 (1974) 557.

[29] W. Nörenberg, Phys. Lett. B 31 (1970) 621.

[30] W. Nörenberg, Phys. Rev. C 5 (1972) 2020.

[31] V.M. Strutinsky, Nucl. Phys. A 95 (1967) 420.

[32] J.O. Rasmussen, W. Nörenberg and H.J. Mang, Nucl. Phys. A 136 (1969) 465.

[33] B.D. Wilkins, E.P. Steinberg and R.R. Chasman, Phys. Rev. C 14 (1976) 1832.

[34] W.D. Myers, Droplet Model of Atomic Nuclei (Plenum, New-York, 1977).

[35] M. Dahlinger, D. Vermeulen and K.H. Schmidt, Nucl. Phys. A 376 (1982) 94.

[36] P. Möller and J.R. Nix, Nucl. Phys. A 361 (1981) 117.

[37] G. Royer and B. Remaud, J. Phys. G 10 (1984) 1541.

[38] P. Möller and J.R. Nix, Proc. Conference on Physics and Chemistry of Fission, Rochester 1974, vol 1 (IAEA Vienna, 1974) p 1.03.

[39] S. Bjornholm and J.E. Lynn, Rev. Mod. Phys 52 (1980) 725.

[40] G. Royer and F. Haddad, Phys. Rev. C 51 (1995) 2813.

[41] F. Haddad and G. Royer, J. Phys. G 21 (1995) 1357.

[42] A.J. Sierk, Phys. Rev. C 33 (1986) 2039.

[43] U.L. Businaro and S. Gallone, Nuovo Cimento 1 (1955) 1277.

[44] L.G. Sobotka et al, Phys. Rev. Lett 53 (1984) 2004.

[45] G. Royer, R K. Gupta and V. Denisov, Nucl. Phys. A (1997) submitted.

[46] P. Möller, J.R. Nix and A.J. Sierk, Nucl. Phys. A 492 (1989) 349.

[47] W.J. Swiatecki, Three Lectures on Macroscopic Aspects of Nuclear Dynamics, Lawrence Berkeley Laboratory Report LBL 8950 (1979).

[48] N. Carjan and M. Kaplan, Phys. Rev. C 45 (1992) 2185.

[49] N. Carjan and J.M. Alexander, Phys. Rev. C 38 (1988) 1692.

[50] A. Galindo-Uribarri et al, Phys. Rev. Lett 71 (1993) 231.

[51] D. Lafosse et al, Phys. Rev. Lett. 74 (1995) 5186.

[52] B. Haas, Proc. International Symposium on Exotic Nuclear Shapes, Debrecen, 1997, (Heavy-ion Physics, 1997) to be published.

[53] J. Dudek, T. Werner and L.L.Riedinger, Phys. Lett. B 211 (1988) 252.

[54] G. Royer and F. Haddad, Phys. Rev. C 47 (1993) 1302.

[55] G. Royer and F. Haddad, J. Phys. G 21 (1995) 339.

[56] C. Beck and A. Szanto de Toledo, Phys. Rev. C 53 (1996) 1989.

[57] G. Royer, Proc. International Symposium on Large Scale Collective Motion of Atomic Nuclei, Brolo 1996, (World Scientific, 1997) to be published.

[58] R.A. Broglia, C.H. Dasso, H. Esbensen and A. Winther, Nucl. Phys. A 349 (1980) 496.

[59] K. Neergard and V.V. Pashkevich, Nucl. Phys. A 262 (1976) 61.

[60] B. Borderie et al, Z. Phys. A 299 (1981) 263.

[61] J.P. Theobald, P. Heeg and M. Mutterer, Nucl. Phys. A 502 (1989) 343c.

[62] A.V. Ramayya et al, Proc. International Workshop on New Ideas on Clustering in Nuclear and Atomic Physics, Marburg 1997, (Nuovo Cimento 1997) to be published.

[63] R. Trockel et al, Phys. Rev. C 39 (1989) 729.

[64] N. Carjan, A.J. Sierk and J.R. Nix, Nucl. Phys. A 452 (1986) 381.

[65] J. Mignen and G. Royer, J. Phys. G 13 (1987) 987.

[66] J. Mignen and G. Royer, J. Phys. G 16 (1990) L227.

[67] G. Royer, F. Haddad and J. Mignen, J. Phys. G 18 (1992) 2015.

[68] G. Royer and J. Mignen, J. Phys. G 18 (1992) 1781.

[69] S.A. Karamyan et al, Sov. J. Nucl. Phys 5 (1967) 559.

[70] A.T. Hasan et al, Phys. Rev. C 49 (1994) 1031. 
[71] G. Royer, J. Phys. G 21 (1995) 249.

[72] S.J. Sanders, C.Beck et al, private communication.

[73] D. Guerreau, Nuclear Matter and Heavy-ion Collisions, ed M. Soyeur, H. Flocard, B. Tamain and M. Porneuf (Plenum, New-York, 1989) p.187.

[74] M. Louvel et al, Nucl. Phys. A 559 (1993) 137.

[75] G. Peilert, H. Stöcker, W. Greiner, A. Rasenhauer, A. Bohnet and J. Aichelin, Phys. Rev. C 39 (1989) 1402.

[76] L.G. Moretto, D.N. Delis and G.J. Wozniak, Phys. Rev. Lett 71 (1993) 3935.

[77] F. Haddad and G. Royer, J. Phys. G 18 (1992) L153.

[78] G. Royer and F. Haddad, J. Phys. G 20 (1994) L131.

[79] A. Kugler et al, Int. Workshop XXII on Gross Properties of Nuclei and Nuclear Excitation, Hirschegg 1994, ed H. Feldmeier and W. Nörenberg (Darmstadt, GSI 1994), p.125.

[80] H.M. Xu, C.A. Gagliardi and R.E. Tribble, Nucl. Phys. A 569 (1994) 575.

[81] W. Bauer, G.F. Bertsch and H. Schulz, Phys. Rev. Lett 69 (1992) 1888.

[82] B.Jouault, F. Sebille, G. Royer and V. de la Mota, Nucl. Phys. A 591 (1995) 497.

[83] B.Jouault, G. Royer, J.F. Lecolley, F. Sebille and F. Haddad, Nucl. Phys. A 615 (1997) 82.

[84] D. Durand, Ganil symposium 'Advances in nuclear physics', Le Pradet, France, (1995).

[85] J.F. Lecolley et al, Phys.Lett. B 387 (1996) 460.

[86]C. Fauchard and G. Royer, Nucl. Phys. A 598 (1996) 125.

[87] G. Royer, F. Haddad and B. Jouault, Nucl. Phys. A 605 (1996) 403.

[88] G. Royer, C. Normand and E. Druet, Nucl. Phys. A (1997) to be submitted. 